\title{
INVERSE SCATTERING WITH PARTIAL DATA ON ASYMPTOTICALLY HYPERBOLIC MANIFOLDS
}

\author{
RAPHAEL HORA AND ANTÔNIO SÁ BARRETO
}

\begin{abstract}
We prove that the scattering matrix at all energies restricted to an open subset of the boundary determines an asymptotically hyperbolic manifold modulo isometries that are equal to the identity on the open subset where the scattering matrix is known.
\end{abstract}

\section{INTRODUCTION}

As the name suggests, asymptotically hyperbolic manifolds are modeled by the hyperbolic space at infinity. The ball model of the hyperbolic space is given by

$$
\stackrel{\circ}{X}=\mathbb{B}^{n+1}=\left\{z \in \mathbb{R}^{n+1}:|z|<1\right\} \text { equipped with the metric } g=\frac{4 d z^{2}}{\left(1-|z|^{2}\right)^{2}} .
$$

We replace $\overline{\mathbb{B}^{n+1}}$ with a $C^{\infty}$, connected, compact manifold $X$ with boundary $\partial X$, of dimension $n+1$. We pick a function $x \in C^{\infty}(X)$ such that $x>0$ in the interior of $X,\{x=0\}=\partial X$, and $d x \neq 0$ at $\partial X$. Such a function will be called a boundary defining function. In the model above $x=1-|z|^{2}$. If $g$ is a Riemannian metric on the interior of $X$ such that

$$
x^{2} g=H
$$

where $H$ is non-degenerate up to $\partial X$, then according to 34 $g$ is complete and its sectional curvatures approach $|d x|_{H}$, as $x \downarrow 0$. In particular, when

$$
|d x|_{H}=1 \text { at } \partial X
$$

the sectional curvatures converge to -1 at the boundary. Such a Riemannian manifold $(X, g)$ for which (1.1) and (1.2) hold is called asymptotically hyperbolic. We are interested in studying the long time behavior of solutions of the wave equation on asymptotically hyperbolic manifolds, and the behavior of the metric, and hence of its geodesics, near $\partial X$ influences how waves scatter.

Notice that $g$ does not determine $H$ and it is interesting to inquire about the behavior of $H=x^{2} g$ at $\partial X$. Since any two defining functions of $\partial X, x$ and $\tilde{x}$, satisfy $x=e^{\omega} \tilde{x}$, with $\omega \in C^{\infty}(X)$, the corresponding $H$ and $\widetilde{H}$ must satisfy $\left.H\right|_{\partial X}=\left.e^{2 \omega(0, y)} \widetilde{H}\right|_{\partial X}$. Hence $\left.H\right|_{\partial X}$ is determined up to a conformal factor which depends on the choice of $x$. We recall the construction of boundary normal coordinates for $g$ in this setting given by Graham [11]. We have $H=x^{2} g=e^{2 \omega} \tilde{x}^{2} g$, and hence $H=e^{2 \omega} \widetilde{H}$. Since $d x=e^{\omega}(\tilde{x} d \omega+d \tilde{x})$, we have

$$
|d x|_{H}^{2}=|d \tilde{x}+\tilde{x} d \omega|_{\widetilde{H}}^{2}=|d \tilde{x}|_{\widetilde{H}}^{2}+\tilde{x}^{2}|d \omega|_{\widetilde{H}}^{2}+2 \tilde{x}\left(\nabla_{\widetilde{H}} \tilde{x}\right) \omega
$$

Hence,

$$
|d x|_{H}=1 \text { if and only if } 2\left(\nabla_{\widetilde{H}} \tilde{x}\right) \omega+\tilde{x}|d \omega|_{\widetilde{H}}^{2}=\frac{1-|d \tilde{x}|_{\widetilde{H}}^{2}}{\tilde{x}},\left.\omega\right|_{\partial X}=0 .
$$


Since by assumption $|d \tilde{x}|_{\widetilde{H}}=1$ at $\partial X$, this is a non-characteristic ODE, and hence it has a solution in a neighborhood of $\partial X$. So we conclude that fixed a representative $h_{0}$ of the conformal class of $\left.H\right|_{\partial X}$, there exists $\epsilon>0$, a neighborhood $U_{\varepsilon}$ of $\partial X$ and a map $\Psi:[0, \epsilon) \times \partial X \longrightarrow U_{\varepsilon}$ such that

$$
\Psi^{*} g=\frac{d x^{2}}{x^{2}}+\frac{h(x, y, d y)}{x^{2}}, \quad h_{0}=h(0, y, d y)
$$

where $h$ is a one-parameter family of metrics on the boundary $\partial X$. This construction works equally well in a neighborhood of an open subset $\Gamma \subset \partial X$.

The spectrum of the Laplacian for this type of manifolds was studied by Mazzeo and Mazzeo and Melrose in [32, 33, 34] and more recently by Bouclet [2]. The spectrum of $\Delta_{g}$, denoted by $\sigma\left(\Delta_{g}\right)$ consists of a finite point spectrum $\sigma_{p p}\left(\Delta_{g}\right)$ and an absolutely continuous spectrum $\sigma_{a c}\left(\Delta_{g}\right)$ satisfying

$$
\sigma_{a c}\left(\Delta_{g}\right)=\left[\frac{n^{2}}{4}, \infty\right), \quad \sigma_{p p}\left(\Delta_{g}\right) \subset\left(0, \frac{n^{2}}{4}\right) .
$$

It follows from (1.4) and the spectral theorem that if $\operatorname{Im} \lambda<<0$, the resolvent for $\Delta_{g}$, denoted by

$$
R(\lambda)=\left(\Delta_{g}-\lambda^{2}-\frac{n^{2}}{4}\right)^{-1}
$$

is a bounded operator in $L^{2}(X)$. The continuation of $R(\lambda)$, as an operator

$$
R(\lambda): C_{0}^{\infty}(\stackrel{\circ}{X}) \longrightarrow C^{-\infty}(\stackrel{\circ}{X}),
$$

from $\operatorname{Im} \lambda<<0$ to the complex plane was first studied by Mazzeo and Melrose in [34], later by Guillarmou [6] and more recently by Vasy [43]

The structure of the generalized eigenfunctions and the analogue of the Sommerfeld radiation condition was studied in [35, 22], where it was proved that for all $f \in C^{\infty}(\partial X)$ and $\lambda \in \mathbb{R} \backslash\{0\}$, there exists a unique $v(z, \lambda) \in C^{\infty}(\stackrel{\circ}{X}), z=(x, y)$ near $\partial X$, satisfying

$$
\begin{gathered}
\left(\Delta_{g}-\lambda^{2}-\frac{n^{2}}{4}\right) v=0 \quad \text { in } \stackrel{\circ}{X} \\
v=x^{i \lambda+n / 2} F_{+}+x^{-i \lambda+n / 2} F_{-}, \quad F_{ \pm} \in C^{\infty}(X),\left.\quad F_{+}\right|_{\partial X}=f .
\end{gathered}
$$

The scattering matrix at energy $\lambda \neq 0$ is defined as the operator

$$
\begin{gathered}
\mathcal{A}(\lambda): C^{\infty}(\partial X) \rightarrow C^{\infty}(\partial X), \\
\left.f \longmapsto F_{-}\right|_{\partial X} .
\end{gathered}
$$

This definition depends on the choice of the function $x$. One can define $\mathcal{A}(\lambda)$ invariantly by making it act on a certain density bundle, but we prefer to fix one function $x$ for which (1.3) holds near $\partial X$ and work with $\mathcal{A}(\lambda)$ as in (1.7). Notice that in view of the construction of $x$, we are implicitly fixing a conformal representative of $\partial X$.

The scattering matrix is the analogue of the Dirichlet-to-Neumann map when $(X, g)$ is a $C^{\infty}$ compact Riemannian manifold with boundary $\partial X$. In this case the Laplacian $\Delta_{g}$ is an elliptic differential operator and it is well known that given $f \in C^{\infty}(\partial X)$, there exists a unique $u \in C^{\infty}(X)$ such that $\Delta_{g} u=0$ in $X$ and $u=f$ at $\partial X$. If $(x, y)$ are geodesic normal coordinates to $\partial X$, one can show that the entire Taylor series of $u(x, y)$ at $\partial X=\{x=0\}$ is determined by the first two terms, $u(0, y)=f(y)$ and $\partial_{x} u(0, y)$, and by the equation. However, since the solution is unique, $\partial_{x} u(0, y)$ is 
globally determined by the equation and the Dirichlet data $f$. The map $\Lambda_{g}: C^{\infty}(\partial X) \longrightarrow C^{\infty}(\partial X)$ defined by $\Lambda_{g} f=\left.\partial_{x} u\right|_{\partial X}$ is called the Dirichlet-to-Neumann map.

The scattering matrix in this class of manifolds was studied in [10, 35, 22, 12, 6]. According to [35, 22], fixed $\lambda \in \mathbb{R}, \backslash 0$ and $x$, the operator $\mathcal{A}(\lambda)$ is pseudodifferential of complex order $2 i \lambda$ with principal symbol

$$
\sigma_{0}(\mathcal{A}(\lambda))(y, \eta)=2^{-i \lambda} \frac{\Gamma(-i \lambda)}{\Gamma(i \lambda)}|\eta|_{h_{0}}^{2 i \lambda}
$$

where $\Gamma$ is the gamma function and $|\eta|_{h_{0}}$ is the length of the co-vector $\eta$ induced by $h_{0}$.

The inverse problem of obtaining information about a compact manifold $(X, g)$ from the Dirichletto-Neumann map $\Lambda_{g}$ has been extensively studied, see for example [41] for a survey about this question. Joshi and Sá Barreo 21] first studied the inverse problem of determining an asymptotically hyperbolic manifold $(X, g)$ from the scattering matrix $\mathcal{A}(\lambda)$ at a fixed energy $\lambda \in \mathbb{R} \backslash 0$, and they showed that $\mathcal{A}(\lambda)$ determines the Taylor series of $h(x, y, d y)$ at $x=0$. More precisely, they proved:

Theorem 1.1. ([21]) Let $(X, g)$ be an asymptotically hyperbolic manifold, let $x$ be a boundary defining function such that (1.3) holds, and let $\mathcal{A}(\lambda)$ be the scattering matrix defined in (1.7) for $\lambda \neq 0$. Let $p \in \partial X$ and let $U \subset \partial X$ be an open subset with $p \in U$, and $a(\lambda, y, \eta)$ be the full symbol of $\mathcal{A}(\lambda)$ with $(y, \eta) \in T^{*} U \backslash 0$. Then there exists a discrete set $\mathcal{Q} \subset \mathbb{R}$ such that if $\lambda \in \mathbb{R} \backslash \mathcal{Q}$, the Taylor series of the tensor $h(x, y, d y)$ at $x=0$, for $y \in U$ is determined by $a(\lambda, y, \eta)$.

Much more can be said in the case when $\mathcal{A}(\lambda)$ is known for every $\lambda \in \mathbb{R} \backslash 0$. The main result of [37] is

Theorem 1.2. ([37]) Let $\left(X_{1}, g_{1}\right)$ and $\left(X_{2}, g_{2}\right)$ be asymptotically hyperbolic manifolds and suppose that $\partial X_{1}=\partial X_{2}=M$ (as manifolds). Let $x_{j} \in C^{\infty}\left(X_{j}\right), j=1,2$, be a defining function of $\partial X_{j}$ for which (1.3) holds, and let $A_{j}(\lambda), j=1,2, \lambda \in \mathbb{R} \backslash\{0\}$, be the corresponding scattering matrices. Suppose that $A_{1}(\lambda)=A_{2}(\lambda)$ for every $\lambda \in \mathbb{R} \backslash\{0\}$. Then there exists a diffeomorphism $\Psi: X_{1} \rightarrow X_{2}$, smooth up to $M$, such that

$$
\Psi=I d \text { on } M \quad \text { and } \quad \Psi^{*} g_{2}=g_{1} .
$$

This problem is related to the question of reconstructing a compact Riemannian manifold with boundary from the Dirichlet-to-Neumann map for the wave equation that was first solved by Belishev and Kurylev [1, using the Boundary Control Method, and a unique continuation result later proved by Tataru [39]. Different proofs, which also rely on the result of Tataru, were given in $[24,25$. The main idea of the proof of Theorem 1.2 was to study the scattering matrix in terms of the wave equation, using Friedlander radiation fields, and adapt the Boundary Control Method of Belishev and Kurylev [1] and Tataru [39] to this setting.

Our goal in this paper is to prove the analogue of Theorem 1.2 when $\mathcal{A}(\lambda)$ is known only on an open set of $\Gamma \subset \partial X$. We define the restriction of $\mathcal{A}(\lambda)$ to $\Gamma$ as the operator

$$
\begin{gathered}
\mathcal{A}_{\Gamma}(\lambda): C_{0}^{\infty}(\Gamma) \longrightarrow C^{\infty}(\Gamma) \\
\left.f \longmapsto(\mathcal{A}(\lambda) f)\right|_{\Gamma} .
\end{gathered}
$$

Our main result is the following

Theorem 1.3. Let $\left(X_{1}, g_{1}\right)$ and $\left(X_{2}, g_{2}\right)$ be asymptotically hyperbolic manifolds and suppose there exists an open set $\Gamma \subset\left(\partial X_{1} \cap \partial X_{2}\right)$ (as manifolds) such that $\partial X_{j} \backslash \Gamma$ do not have empty interior, for $j=1,2$. Let $x_{j} \in C^{\infty}\left(X_{j}\right), j=1,2$, be a defining function of $\partial X_{j}$ for which (1.3) holds, and 
let $\mathcal{A}_{j, \Gamma}(\lambda), j=1,2, \lambda \in \mathbb{R} \backslash\{0\}$, be the corresponding scattering matrices restricted to $\Gamma$. Suppose that $\mathcal{A}_{1, \Gamma}(\lambda)=\mathcal{A}_{2, \Gamma}(\lambda)$ for every $\lambda \in \mathbb{R} \backslash\{0\}$. Then there exists a diffeomorphism $\Psi: X_{1} \rightarrow X_{2}$, smooth up to $\partial X_{1}$, such that

$$
\Psi=I d \text { on } \Gamma \quad \text { and } \quad \Psi^{*} g_{2}=g_{1} .
$$

The reconstruction of a compact manifold in the case where the Dirichlet-to-Neumann map is only known on part of the boundary was carried out by Kurylev and Lassas [26] using a modification of the Boundary Control Method. As in the proof of Theorem 1.2, we will adapt the Boundary Control Methods to this setting by using the dynamical formulation of the scattering matrix in terms of Friedlander radiation fields.

Equation (1.4) gives a decomposition of $L^{2}(X)$

$$
L^{2}(X)=L_{p p}^{2}(X) \oplus L_{a c}^{2}(X),
$$

where $L_{p p}^{2}(X)$ is the finite dimensional space spanned by the eigenfunctions of $\Delta_{g}$ and $L_{a c}^{2}(X)$ is the orthogonal complement of $L_{p p}^{2}(X)$. It follows from Corollary 6.3 of [37] that

$$
C_{0}^{\infty}(\stackrel{\circ}{X}) \cap L_{a c}^{2}(X) \text { is dense in } L_{a c}^{2}(X) .
$$

Let $u$ satisfy the wave equation

$$
\begin{gathered}
\left(D_{t}^{2}-\Delta_{g}+\frac{n^{2}}{2}\right) u(t, z)=0 \quad \text { on } \mathbb{R}_{+} \times \stackrel{\circ}{X}, \\
u(0, z)=f_{1}(z), \quad D_{t} u(0, z)=f_{2}(z), \quad f_{1}, f_{2} \in C_{0}^{\infty}(\stackrel{\circ}{X}) .
\end{gathered}
$$

This equation has a conserved energy given by

$$
\begin{gathered}
E\left(u, \partial_{t} u\right)(t)=\int_{X}\left(|d u(t)|^{2}-\frac{n^{2}}{4}|u(t)|^{2}+\left|\partial_{t} u(t)\right|^{2}\right) d \operatorname{vol}_{g}, \\
E\left(u, \partial_{t} u\right)(0)=E\left(f_{1}, f_{2}\right)=\int_{X}\left(\left|d f_{1}\right|^{2}-\frac{n^{2}}{4}\left|f_{1}\right|^{2}+\left|f_{2}\right|^{2}\right) d \operatorname{vol}_{g},
\end{gathered}
$$

$E\left(f_{1}, f_{2}\right)$ is positive only when projected onto $L_{a c}^{2}(X)$. As in [37], we define the energy space

$$
H_{E}(X)=\left\{\left(f_{1}, f_{2}\right): f_{1}, f_{2} \in L^{2}(X), d f_{1} \in L^{2}(X) \text { and } E\left(f_{1}, f_{2}\right)<\infty\right\}
$$

and the projector

$$
\begin{gathered}
\mathcal{P}_{a c}: L^{2}(X) \longrightarrow L_{a c}^{2}(X) \\
f \longmapsto f-\sum_{j=1}^{N}\left\langle f, \phi_{j}\right\rangle \phi_{j},
\end{gathered}
$$

where $\left\{\phi_{j}, 1 \leq j \leq N\right\}$ are the eigenfunctions of $\Delta_{g}$. Let

$$
E_{a c}(X)=\mathcal{P}_{a c}\left(H_{E}(X)\right) .
$$

The wave group induces a strongly continuous group of unitary operators:

$$
\begin{gathered}
U(t): E_{a c}(X) \longrightarrow E_{a c}(X) \\
\left(f_{1}, f_{2}\right) \longmapsto\left(u(t), \partial_{t} u(t)\right) .
\end{gathered}
$$


Following Friedlander [4, 5], the radiation fields for asymptotically hyperbolic manifolds were defined in [37]. It was shown in [37] that if $x$ is a boundary defining function of $\partial X$ for which (1.3) holds, and $\left(f_{1}, f_{2}\right) \in C_{0}^{\infty}(X) \cap E_{a c}(X)$, then

$$
V_{+}(x, s, y)=x^{-n / 2} u(s-\log x, x, y) \in C^{\infty}\left([0, \varepsilon)_{x} \times \mathbb{R}_{s} \times \partial X\right),
$$

and the forward radiation field is defined as the map

$$
\begin{gathered}
\mathcal{R}_{+}: C_{0}^{\infty}(\stackrel{\circ}{X}) \times C_{0}^{\infty}(\stackrel{\circ}{X}) \longrightarrow C^{\infty}(\mathbb{R} \times \partial X), \\
\mathcal{R}_{+}\left(f_{1}, f_{2}\right)(s, y)=D_{s} V_{+}(0, s, y)=\lim _{x \downarrow 0} x^{-\frac{n}{2}} D_{s} u(s-\log x, x, y) .
\end{gathered}
$$

Similarly, if one considers the behavior of $u$ for $t<0$, again with the initial data $\left(f_{1}, f_{2}\right) \in$ $C_{0}^{\infty} \cap E_{a c}(X)$, then

$$
V_{-}(x, s, y)=x^{-n / 2} u(s+\log x, x, y) \in C^{\infty}\left([0, \varepsilon)_{x} \times \mathbb{R}_{s} \times \partial X\right),
$$

and thus defines the backward radiation field

$$
\begin{aligned}
\mathcal{R}_{-}: & C_{0}^{\infty}(\stackrel{\circ}{X}) \times C_{0}^{\infty}(\stackrel{\circ}{X}) \longrightarrow C^{\infty}(\mathbb{R} \times \partial X), \\
& \mathcal{R}_{-}\left(f_{1}, f_{2}\right)(s, y)=D_{s} V_{-}(0, s, y) .
\end{aligned}
$$

It was shown in [37] that the maps $\mathcal{R}_{ \pm}$extend to unitary operators

$$
\begin{gathered}
\mathcal{R}_{ \pm}: E_{a c}(X) \longrightarrow L^{2}(\mathbb{R} \times \partial X) \\
\left(f_{1}, f_{2}\right) \longmapsto \mathcal{R}_{ \pm}\left(f_{1}, f_{2}\right),
\end{gathered}
$$

which are translation representations of the wave group as in the Lax-Phillips theory [31], i.e.

$$
\mathcal{R}_{ \pm}\left(U(T)\left(f_{1}, f_{2}\right)\right)(s, y)=\mathcal{R}_{ \pm}\left(f_{1}, f_{2}\right)(s+T, y) .
$$

One can define the scattering operator

$$
\begin{gathered}
\mathcal{S}: L^{2}(\mathbb{R} \times \partial X) \longrightarrow L^{2}(\mathbb{R} \times \partial X), \\
\mathcal{S}=\mathcal{R}_{+} \circ \mathcal{R}_{-}^{-1},
\end{gathered}
$$

which is unitary in $L^{2}(\partial X \times \mathbb{R})$ and commutes with translations in view of (1.17). The results of [21] and [8] were used in [37] to show that the stationary and dynamical definitions of the scattering matrix are equivalent. If $\mathcal{A}(\lambda)$ is defined by (1.7) and $\mathcal{S}$ is defined by (1.18), then

$$
\mathcal{A}=\mathcal{F S F}^{-1},
$$

where $\mathcal{F}$ denotes the partial Fourier transform in the variable $s$.

The relationship between $\mathcal{R}_{ \pm}$and $\mathcal{A}(\lambda)$ was made more explicit in [37], where it was shown (see equation (6.12) of [37]) that if one takes the partial Fourier transform of $\mathcal{R}_{+}$with respect to the variable $s$, then if one denotes

$$
\mathcal{F}\left(\mathcal{R}_{+}(0, h)\right)(\lambda, y)=i \lambda \int_{X} E\left(\frac{n}{2}+i \lambda, y, z\right) h(z) d \operatorname{vol}_{g}(z),
$$

then for any $f \in C^{\infty}(\partial X)$,

$$
u(z)=\int_{\partial X} E\left(\frac{n}{2}+i \lambda, y^{\prime}, z\right) f\left(y^{\prime}\right) d \operatorname{vol}_{h_{0}}\left(y^{\prime}\right)
$$


satisfies

$$
\begin{gathered}
\left(\Delta_{g}-\frac{n^{2}}{4}-\lambda^{2}\right) u=0, \\
u(x, y)=x^{\frac{n}{2}+i \lambda} F_{+}+x^{\frac{n}{2}-i \lambda} F_{-}, \text {where }\left.F_{+}\right|_{\partial X}=f,\left.\quad F_{-}\right|_{\partial X}=\mathcal{A}(\lambda) f .
\end{gathered}
$$

1.1. Acknowledgements. Hora and Sá Barreto are grateful to the NSF for the support provided under grant DMS-0901334.

\section{The Local Support Theorem}

The purpose of this section is to establish a relationship between the support of $\mathcal{R}_{+}(0, f)$ and the support of $f$. Again, it is useful to recall the analogy with the Dirichlet-to-Neumann map on compact manifolds with boundary. If $(X, g)$ is a compact Riemannian manifold with boundary, the finite speed of propagation for the wave equation implies that if $\Gamma \subset \partial X$ is an open subset of the boundary and $u(t, z)$ is a solution to the Dirichlet problem for the wave equation

$$
\begin{gathered}
\left(\partial_{t}^{2}+\Delta_{g}\right) u=0 \text { in }(-T, T) \times \stackrel{\circ}{X} \\
u(0, z)=0, \quad \partial_{t} u(0, z)=f(z) \in C^{\infty}(\stackrel{\circ}{X}), \\
\left.u(t, z)\right|_{(-T, T) \times \partial X}=0
\end{gathered}
$$

then the normal derivative $\left.\partial_{\mu} u(t, z)\right|_{\left(-T^{\prime}, T^{\prime}\right) \times \partial X}=0$, provided $T^{\prime} \in(0, T), z \in \bar{\Gamma}$ and $d_{g}(\operatorname{Supp} f, \bar{\Gamma})<$ $T^{\prime}$, where $d_{g}$ denotes the distance with respect to the metric $g$. The converse of this result holds, and is a consequence of a theorem of Tataru [39], see also [20, 36, 40].

In the current setting, the manifold is not compact and the distance from a point in the interior of $X$ to the boundary is infinite. If $x$ is chosen such that the metric $g$ is in the form (1.3), the corresponding Lorentzian metric takes the form

$$
G_{L}=d t^{2}-\frac{d x^{2}}{x^{2}}-\frac{h(x, y, d y)}{x^{2}} .
$$

From the construction of the defining function $x$ for which (1.3) holds from [1] (see section 11), given two points along the curves normal to $\Gamma$, the distance between $(x, y)$ and $(\alpha, y)$, with $\alpha<x$ small enough, is $-\log \left(\frac{x}{\alpha}\right)$, and therefore, if time measures the arc-length along these geodesics, one has $s=t+\log x=\log \alpha$. In the case of data given on the whole boundary, the following support theorem was proved in [37]:

Theorem 2.1. A function $f \in L_{a c}^{2}(X)$ is such that $\mathcal{R}_{+}(0, f)(s, y)=0$ for every $s \leq s_{0}<<0$ and $y \in \partial X$ if and only if $f(x, y)=0$ if $x \leq e^{s_{0}}, y \in \partial X$.

One should remark that the fact this result is true for $f \in L_{a c}^{2}(X)$ is particular to the hyperbolic setting. For instance, the analogue of this result is not true on Euclidean space; in that case one needs to assume that $f$ is rapidly decaying, i.e. $f$ vanishes to infinite order at $\partial X$. In the case where $(X, g)$ is the hyperbolic space, this result is due to Lax and Phillips [27, 28, 29, see also the work of Helgason [13, 14, 15, 16. Our goal for this section is to prove the local version of this result. First we observe that if $\Gamma \subset \partial X$ is an open subset, the construction of the defining function works just as well if one restricts it to $\Gamma$. We will prove

Theorem 2.2. Let $\Gamma \subset \partial X$ be an open subset and $s_{0} \in \mathbb{R}$. A function $f \in L_{a c}^{2}(X)$ is such that $\mathcal{R}_{+}(0, f)(s, y)=0$ for every $s \leq s_{0}$ and $y \in \bar{\Gamma}$ if and only if there exists $\varepsilon>0$ such that $f=0$ a.e. 
in the set

$$
\mathcal{D}_{s_{0}}(\Gamma)=\left\{z \in X: \exists w=\left(\alpha, y^{\prime}\right) \text { with } 0<\alpha<\varepsilon \leq e^{s_{0}} \text { and } y^{\prime} \in \Gamma, \quad d_{g}(z, w)<\log \left(\frac{e^{s_{0}}}{\alpha}\right)\right\},
$$

where $d_{g}$ denotes the distance function with respect to the metric $g$.

Notice that $\mathcal{D}_{s_{0}}(\Gamma)$ is equal to the union of open balls centered at $\left(\alpha, y^{\prime}\right)$, with $0<\alpha<\varepsilon$, and $y^{\prime} \in \Gamma$, and radii $\log \left(\frac{e^{s_{0}}}{\alpha}\right)$ measured with respect to the metric $g$. Therefore $\mathcal{D}_{s_{0}}(\Gamma)$ is an open subset of $\stackrel{\circ}{X}$.

In Theorem 2.1 one assumes that $s_{0}<<0$ and the conclusion about the support of $f$ is expressed in terms of coordinates $(x, y)$, which are only valid in a neighborhood of $\partial X$. There is no restriction on $s_{0}$ in Theorem 2.2, so it is a generalization of Theorem 2.1 in this sense as well. On the other hand, if $\Gamma=\partial X$ and $s_{0}<<0$ is such that coordinates $(x, y)$ hold in $(0, \varepsilon) \times \partial X$, with $e^{s_{0}}<\varepsilon$, then $\mathcal{D}_{s_{0}}(\partial X)=\left\{(x, y): x<e^{s_{0}}, y \in \partial X\right\}$. Indeed, given a point $z=(x, y)$ with $x<e^{s_{0}}$, if $0<\alpha<x$, $d_{g}((\alpha, y),(x, y))=\log \left(\frac{x}{\alpha}\right)<\log \left(\frac{e^{s_{0}}}{\alpha}\right)$. But, if $\varepsilon>x>e^{s_{0}}$, and $z=\left(x, y^{\prime}\right)$, then for any $w=(\alpha, y)$, with $\alpha<e^{s_{0}}, d_{g}(z, w)>\log \left(\frac{e^{s_{0}}}{\alpha}\right)$. So $z \notin \mathcal{D}_{s_{0}}(\partial X)$.

Lax and Phillips [30] also proved this result when $(X, g)$ is the hyperbolic space. It is useful to explain what the set $\mathcal{D}_{s_{0}}(\Gamma)$ is when $(X, g)$ is the hyperbolic space. It is easier to do the computations for the half-space model of hyperbolic space which is given by

$$
\mathbb{H}^{n+1}=\left\{(x, y): \quad x>0, \quad y \in \mathbb{R}^{n}\right\}, \text { and the metric } g=\frac{d x^{2}+d y^{2}}{x^{2}} .
$$

The distance function between $z=(x, y)$ and $w=\left(\alpha, y^{\prime}\right)$ satisfies

$$
\cosh d_{g}(z, w)=\frac{x^{2}+\alpha^{2}+\left|y-y^{\prime}\right|^{2}}{2 x \alpha} .
$$

Since $d_{g}\left(z, z^{\prime}\right) \leq \log \left(\frac{e^{s_{0}}}{\alpha}\right)$, we obtain

$$
\left(x-\frac{1}{2} e^{s_{0}}\left(1+\alpha^{2} e^{-2 s_{0}}\right)\right)^{2}+\left|y-y^{\prime}\right|^{2} \leq \frac{1}{4} e^{2 s_{0}}\left(1+\alpha^{2} e^{-2 s_{0}}\right)^{2}-\alpha^{2}=\frac{1}{4} e^{2 s_{0}}\left(1-\alpha^{2} e^{-2 s_{0}}\right)^{2},
$$

which corresponds to a ball $D(\alpha)$ centered at $\left(\frac{1}{2} e^{s_{0}}\left(1+\alpha^{2} e^{-2 s_{0}}\right), y^{\prime}\right)$ and radius $\frac{1}{2} e^{s_{0}}\left(1-\alpha^{2} e^{-2 s_{0}}\right)$. Since $\alpha<e^{s_{0}}$, we have $D(\alpha) \subset D(0)$, as shown in figure1. This ball is tangent to the plane $x=e^{s_{0}}$ at the point $\left(e^{s_{0}}, y^{\prime}\right)$. When $\alpha=0$ the ball $D(0)$ has center $\left(\frac{1}{2} e^{s_{0}}, y^{\prime}\right)$ and radius $\frac{1}{2} e^{s_{0}}$ and is also tangent to the plane $\{x=0\}$. The boundary of $D(0)$ is called a horosphere since it is orthogonal to the geodesics emanating from the point $\left(0, y^{\prime}\right)$. When $\alpha=e^{s_{0}}, D\left(e^{s_{0}}\right)=\left(e^{s_{0}}, y^{\prime}\right)$.

The set $\mathcal{D}_{s_{0}}(\Gamma)$ consists of the union of horospheres with radii $\frac{1}{2} e^{s_{0}}$ tangent to points $\left(0, y^{\prime}\right)$ with $y^{\prime} \in \Gamma$, see figure 2. In the case of $\mathbb{H}^{n-1}$, the radiation field is given in terms of the horocyclic Radon transform. The result of Lax and Phillips says that if the integral of $f$ over all horospheres tangent to points $(0, y)$, with $y \in \Gamma$ and radii less than or equal to $\frac{1}{2} e^{s_{0}}$, then $f=0$ in the region given by the union of these horocycles.

It is interesting to explain Theorem 2.2 in terms of sojourn times in the case where $(X, g)$ is nontrapping. In this setting the sojourn time plays the role of the distance function to the boundary of $X$ and is closely related to the Busemann function used in differential geometry. The sojourn times for non-trapping asymptotically hyperbolic manifolds was studied in [38]. Let $g^{*}$ denote the principal symbol of the Laplacian with respect to $g$. In local coordinates (1.3),

$$
g^{*}=x^{2} \xi^{2}+x^{2} h(x, y, \eta)
$$




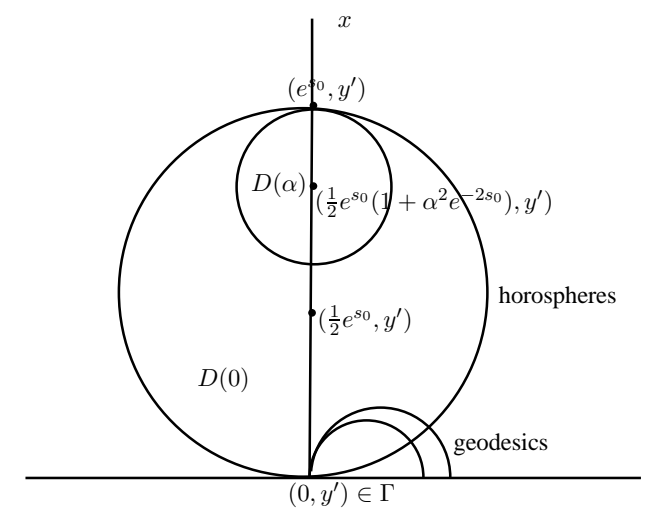

FiguRE 1. The horospheres tangent at $\left(0, y^{\prime}\right)$ and the balls $D(\alpha)$
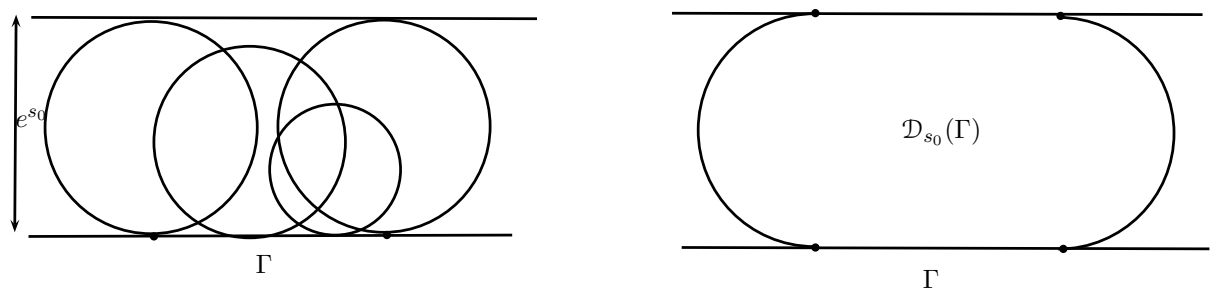

Figure 2. The set $\mathcal{D}_{s_{0}}(\Gamma)$ when $(X, g)$ is the hyperbolic space is given by the union of horospheres tangent to points on $\Gamma$ and radii less than or equal to $\frac{1}{2} e^{s_{0}}$.

where $h$ is the principal symbol of $\Delta_{h}$ as a differential operator on $\partial X$. The set of points

$$
S^{*} \stackrel{\circ}{X}=\left\{q=(z, \zeta) \in T^{*} \stackrel{\circ}{X}: g^{*}(q)=1\right\}
$$

is the unit co-sphere bundle in $T^{*} X$ with respect to $g^{*}$. A integral curve of the Hamilton vector field of $g^{*}$ is called a bicharacteristic. These curves are parametrized by time $t$ and are denoted by $\exp \left(t H_{g^{*}}\right)(q)$, and since $H_{g^{*}}$ is tangent to the level surfaces of $g^{*}, t$ is the arc-length. It is well known that if $\pi_{1}$ denotes the canonical projection $\pi_{1}: T^{*} \stackrel{\circ}{X} \longrightarrow \stackrel{\circ}{X}$, then $\pi_{1}(\gamma(t))$ is a geodesic of the metric $g$ in $X$ passing through $z$, the projection of the point $q$. We say that a bicharacteristic is not trapped for positive (negative) times, if its projection to $\stackrel{\circ}{X}$ leaves any compact set $K \subset \stackrel{\circ}{X}$ in finite time as $t \rightarrow \infty(-\infty)$. The manifold $(X, g)$ is non-trapping if every bicharacteristic is non-trapped for positive and negative times. In this case, it was shown in 38] that the following functions are well defined

$$
\begin{gathered}
s(q)=\lim _{t \rightarrow \infty}\left(t+\log \left[x\left(\exp \left(t H_{g^{*}}\right)(q)\right)\right]\right) \text { and } \\
y=\lim _{t \rightarrow \infty} y\left(\exp \left(t H_{g^{*}}\right)(q)\right),
\end{gathered}
$$

where $x\left(\exp \left(t H_{g^{*}}\right)(q)\right)$ and $y\left(\exp \left(t H_{g^{*}}\right)(q)\right)$ denote the coordinates (1.3) of the point $\pi_{1}(\gamma(t))$. The function $s(q)$ is called the sojourn time of the bicharacteristic through $q$. In a compact manifold, this would be the analogue of the set of points in the interior whose distance to the boundary is $s$. We have the following consequence of Theorem 2.2 . 
Corollary 2.3. Let $f$ and $\Gamma \subset \partial X$ satisfy the hypotheses of Theorem 2.2 and suppose that $(X, g)$ is non-trapping. Then $f=0$ a.e on the set of points $z \in \stackrel{\circ}{X}$ such that exists a geodesic $\gamma(t)$ parametrized by the arc-length such that $\gamma(t) \rightarrow y \in \Gamma$ as $t \rightarrow \infty$, and

$$
\lim _{t \rightarrow \infty}\left(t+\log (x(\gamma(t)))=s<s_{0}\right.
$$

Proof. Suppose there exists a geodesic $\gamma(t)$, parametrized by the arc-length $t$ such that $\gamma(0)=z$ and $\lim _{t \rightarrow \infty} \gamma(t)=y$, moreover $\lim _{t \rightarrow \infty}\left(t+\log (x(\gamma(t)))=s<s_{0}\right.$. Since $t$ is the arc-length parameter, $d(z,(x(\gamma(t)), y)) \leq t$ and $s<s_{0}$, then there exists $T>0$ such that for $t>T, \gamma(t) \in U \sim[0, \varepsilon) \times \partial X$, where coordinates (1.3) are valid and $t+\log x(\gamma(t))<s_{0}$. Therefore, if $t>T$,

$$
d(z,(x(t), y)) \leq t<s_{0}-\log x(\gamma(t))=\log \left(\frac{e^{s_{0}}}{x(\gamma(t))}\right) .
$$

Hence $z \in \mathcal{D}_{s_{0}}(\Gamma)$.

The proof of Theorem 2.2 will be divided in several steps. We begin by proving the sufficiency of the condition in Theorem 2.2 ,

Lemma 2.4. Let $f \in L_{a c}^{2}(X)$ be such that $f(z)=0$ in the set $\mathcal{D}_{s_{0}}(\Gamma)$. Then $\mathcal{R}_{+}(0, f)(s, y)=0$ if $s \leq s_{0}$ and $y \in \Gamma$.

Proof. Let $u(t, w)$ satisfy the wave equation (1.11) with initial data $(0, f)$. The finite speed of propagation for solutions of the wave equation guarantees that $u(t, w)=0$ if $0 \leq t \leq d_{g}(w, \operatorname{Supp} f)$. In particular, since $f(z)=0$ for $z \in \mathcal{D}_{s_{0}}(\Gamma)$, if $w=(\alpha, y)$ with $y \in \Gamma$, then $u(t, w)=0$ if $0 \leq t \leq$ $\log \left(\frac{e^{s_{0}}}{\alpha}\right)$. Since $s=t+\log x$, when $x=\alpha$ we have that $V_{+}(\alpha, s, y)=x^{-\frac{n}{2}} u(s-\log \alpha, \alpha, y)=0$ provided $0 \leq s-\log \alpha \leq \log \left(\frac{e^{s_{0}}}{\alpha}\right)$. Therefore one has $V_{+}(\alpha, s, y)=0$ provided $\log \alpha \leq s \leq s_{0}$ and $y \in \Gamma$. This implies that $\mathcal{R}_{+}(0, f)(s, y)=0$ if $s \leq s_{0}$ and $y \in \Gamma$.

The proof of the converse relies on delicate unique continuation results. First, it is important to realize that we may assume that $f \in C^{\infty}(\stackrel{\circ}{X})$. Indeed, since $\mathcal{R}(0, f)(s, y)=0$ for $s \leq s_{0}$ and $y \in \Gamma$, we may take the convolution of $\mathcal{R}_{+}(0, f)$ with $\psi_{\delta} \in C_{0}^{\infty}(\mathbb{R})$, even and supported in $(-\delta, \delta)$, with $\int \psi_{\delta}(s) d s=1$, and hence $H_{\delta}(s, y)=\psi_{\delta} * \mathcal{R}_{+}(0, f)=\mathcal{R}\left(0, \widetilde{f}_{\delta}\right)=0$ if $s \leq s_{0}+\delta$, and since for every $k \geq 0$,

$$
\partial_{s}^{2 k} H_{\delta}(s, y)=\mathcal{R}_{+}\left(0,\left(\Delta-\frac{n^{2}}{4}\right)^{k} \widetilde{f}_{\delta}\right) \in L^{2}(\mathbb{R} \times \partial X),
$$

and using that $\mathcal{R}_{+}$is unitary, then $\left(\Delta-\frac{n^{2}}{4}\right)^{k} \widetilde{f}_{\delta} \in L^{2}(X)$. Therefore, by elliptic regularity $\widetilde{f}_{\delta} \in$ $C^{\infty}(\stackrel{\circ}{X})$. If one proves that $\widetilde{f}_{\delta}(z)=0$ for $z \in \mathcal{D}_{s_{0}}(\Gamma)$, is supported in $\left\{x \geq e^{s_{0}+\delta}\right\}$, since $\widetilde{f}_{\delta} \rightarrow f$ as $\delta \rightarrow 0$, it follows that $f(z)=0$ for $z \in \mathcal{D}_{s_{0}}(\Gamma)$.

The next step in the proof is

Proposition 2.5. Let $f \in C^{\infty}(X)$ satisfy the hypotheses of Theorem 2.2. Let u satisfy the initial value problem for the wave equation (1.11) with initial data $(0, f)$, and let $V_{+}(x, s, y)$ be defined as in (1.13). Then, in the sense of distributions, $\partial_{x}^{k} V_{+}(0, s, y)=0, k=0,1, \ldots$, provided $s \leq s_{0}$, and $y \in \Gamma$. Moreover, for every $p \in \Gamma$ there exists $\delta>0$ such that $V_{+}(x, s, y)=0$ if $0<x<\delta$, $|y-p|<\delta$ and $s<\log \delta$. 
Proof. In local coordinates (1.3) and for $s=t+\log x, x \in[0, \varepsilon)$, the wave operator, conjugated by appropriate powers of $x$, can be written as

$$
P=-x^{-\frac{n}{2}-1}\left(D_{t}^{2}-\Delta-\frac{n^{2}}{4}\right) x^{\frac{n}{2}}=\partial_{x}\left(2 \partial_{s}+x \partial_{x}\right)-x \Delta_{h}+A \partial_{s}+A x \partial_{x}+\frac{n}{2} A,
$$

where $\Delta_{h}$ is the Laplace operator on $\partial X$ corresponding to the metric $h(x)$. In local coordinates

$$
\begin{gathered}
\Delta_{h}=-\frac{1}{\sqrt{\theta}} \partial_{y_{i}}\left(\sqrt{\theta} h^{i j} \partial_{y_{j}}\right) \text { where } \\
h=\left(h_{i j}(x, y)\right) \quad h^{-1}=\left(h^{i j}(x, y)\right), \quad \theta=\operatorname{det}\left(h_{i j}\right) \text { and } A=\frac{1}{\sqrt{\theta}} \partial_{x} \sqrt{\theta} .
\end{gathered}
$$

The Cauchy problem (1.11), with initial data $(0, f)$ translates into the following initial value problem for $V(x, s, y)=x^{-\frac{n}{2}} u(s+\log x, x, y)$,

$$
\begin{gathered}
P V(x, s, y)=0 \text { in } \mathbb{R} \times(0, \varepsilon)_{x} \times \partial X \\
V(x, \log x, y)=0, \quad D_{s} V(x, \log x, y)=x^{-\frac{n}{2}} f(x, y) .
\end{gathered}
$$

Since one cannot prove unique continuation results across $x=0$, then as in [37] we have to compactify the space in a suitable way, and instead of working with coordinates $x$ and $s$, it is more convenient to work with the variables

$$
s_{+}=s=t+\log x \text { and } s_{-}=t-\log x .
$$

Since we are interested in the behavior of $V_{+}(x, s, y)$ defined in (1.13) for $s=s_{+} \sim-\infty$, and by parity for $s_{-} \sim \infty$, we introduce the following change of variables

$$
\mu=e^{-\frac{s_{-}}{2}} \text { and } \nu=e^{\frac{s_{+}}{2}} .
$$

This implies that

$$
s=2 \log \nu, \quad x=\mu \nu .
$$

We remark that the change of variables $(t, x, y) \mapsto\left(\frac{\mu+\nu}{2}, \frac{\mu-\nu}{2}, y\right)$, which will be used below, plays the role of the Kelvin transform for the Euclidean wave equation.

In coordinates $(\mu, \nu, y)$, the operator $P$ has the form

$$
\widetilde{P}=\partial_{\mu} \partial_{\nu}-\mu \nu \Delta_{h}+\frac{1}{2} A\left(\mu \partial_{\mu}+\nu \partial_{\nu}\right)+\frac{n}{2} A,
$$

where $h=h(\mu \nu), A=A(\mu \nu, y)$. If $W(\mu, \nu, y)=V_{+}(\mu \nu, 2 \log \nu, y)$, the Cauchy problem (2.4) becomes

$$
\begin{gathered}
\widetilde{P} W=0, \quad \mu, \nu \in(0, \varepsilon), \quad y \in \partial X \\
W(\mu, \mu, y)=0, \quad \partial_{\mu} W(\mu, \mu, y)=-\mu^{-1-n} f\left(\mu^{2}, y\right) .
\end{gathered}
$$

Recall that we are assuming that $f \in C^{\infty}(\stackrel{\circ}{X})$, so $W$ is $C^{\infty}$ in the region $\{\mu>0, \nu>0\}$. The issue here is the behavior of $W$ at $\{\mu=0\} \cup\{\nu=0\}$. One should notice that if $F(\mu, y)=\mu^{-1-n} f\left(\mu^{2}, y\right)$, then

$$
\int_{0}^{\varepsilon} \int_{\partial X} \mu|F(\mu, y)|^{2} \theta^{\frac{1}{2}}\left(\mu^{2}, y\right) d y d \mu=\frac{1}{2} \int_{0}^{\varepsilon^{2}} \int_{\partial X}|f(x, y)|^{2} x^{-n-1} \theta^{\frac{1}{2}}(x, y) d x d y=\frac{1}{2}\|f\|_{L^{2}(X)}^{2}
$$




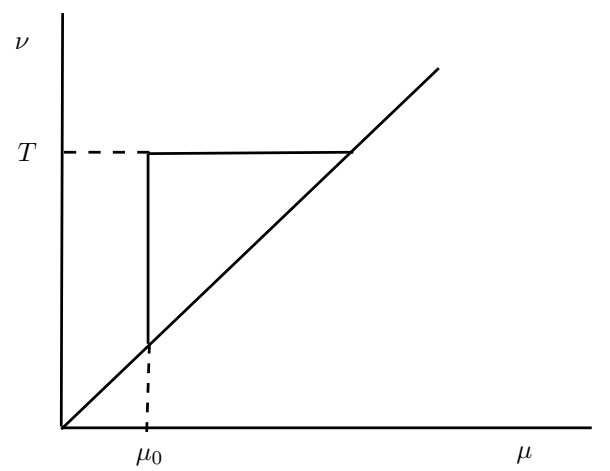

FiguRE 3. The region of integration in (2.9)

We know from Theorem 2.1 of [37] that if $f \in C_{0}^{\infty}(\stackrel{\circ}{X}) \cap L_{a c}^{2}(X)$, then $W$ has a $C^{\infty}$ extension $W(\mu, \nu, y) \in C^{\infty}([0, \varepsilon] \times[0, \varepsilon] \times \partial X)$ up to $\{\mu=0\} \cup\{\nu=0\}$, and since $\partial_{s}=\frac{1}{2}\left(\nu \partial_{\nu}-\mu \partial_{\mu}\right)$, then, provided $f \in C_{0}^{\infty}(\stackrel{\circ}{X}) \cap L_{a c}^{2}(X)$,

$$
\mathcal{R}_{+}(0, f)(2 \log \nu, y)=\left.\frac{1}{2}\left[\left(\nu \partial_{\nu}-\mu \partial_{\mu}\right) W(\mu, \nu, y)\right]\right|_{\mu=0}=\frac{1}{2} \nu \partial_{\nu} W(0, \nu, y),
$$

and we want to show that this restriction makes sense for $f \in L_{a c}^{2}(X)$. We will work in the region $\{\nu \geq \mu\}$, but since the solution to (2.6) is odd under the change $(\mu, \nu) \mapsto(\nu, \mu)$, a similar analysis works for the backward radiation field in the region $\{\nu \leq \mu\}$.

Again, assuming that $f \in C_{0}^{\infty}(\stackrel{\circ}{X}) \cap L_{a c}^{2}(X)$, and $W$ satisfies (2.6), one can combine equations (4.11), (4.14) and (4.15) of [37], and (2.7) to arrive at the following estimate (see figure 3): For $\mu_{0} \in[0, \varepsilon), T \in[0, \varepsilon)$, there exists $C>0$ depending on the operator $\widetilde{P}$ and $\varepsilon$ only, such that

$$
\begin{gathered}
I\left(W, \mu_{0}, T\right)=\left.\int_{\mu_{0}}^{T} \int_{\partial X}\left[\left(|W|^{2}+\mu\left|\partial_{\mu} W\right|^{2}+\mu \nu^{2}\left|d_{h(\mu \nu)} W\right|^{2}\right) \sqrt{\theta}(\mu \nu)\right]\right|_{\nu=T} d y d \mu+ \\
\left.\int_{\mu_{0}}^{T} \int_{\partial X}\left[\left(|W|^{2}+\nu\left|\partial_{\nu} W\right|^{2}+\mu^{2} \nu\left|d_{h(\mu \nu)} W\right|^{2}\right) \sqrt{\theta}(\mu \nu)\right]\right|_{\mu=\mu_{0}} d y d \nu \leq C\|f\|_{L^{2}(X)},
\end{gathered}
$$

If $f \in L_{a c}^{2}(X)$ and if we take a sequence $f_{j} \in C_{0}^{\infty}(\stackrel{\circ}{X}) \cap L_{a c}^{2}(X)$, with $\left\|f-f_{j}\right\|_{L^{2}(X)} \rightarrow 0$, (2.9) shows that fixed $\mu_{0} \in\left[0, \varepsilon_{0}\right)$, then

$$
I\left(W_{j}-W_{k}, \mu_{0}, T\right) \leq C\left\|f_{j}-f_{k}\right\|_{L^{2}(X)},
$$

and in particular, if $\mu_{0} \in[0, \varepsilon)$, and if $W$ is a solution of (2.6) with $f \in L_{a c}^{2}(X)$, then for $\mu_{0} \in[0, \varepsilon)$,

$$
\int_{\mu_{0}}^{T} \int_{\partial X} \nu\left|\partial_{\nu} W\left(\mu_{0}, \nu, y\right)\right|^{2} \sqrt{\theta}\left(\mu_{0} \nu, y\right) d \nu d y \leq C\|f\|_{L^{2}(X)}
$$

is well defined. Since the radiation field is unitary, then in the sense of (2.10) for $\mu_{0}=0$, equation (2.8) holds for $f \in L_{a c}^{2}(X)$.

Next we want to show that, if $\nu \partial_{\nu} W(0, \nu, y)=0$, and by symmetry $\mu \partial_{\mu} W(\mu, 0, y)=0$, then in the sense of distributions, $W$ has an extension across $\{\mu=0\} \cup\{\nu=0\}$ such that $W=0$ for $\mu<0, \nu<0$, with $|\mu|<\varepsilon,|\nu|<\varepsilon$, which remains a solution to (2.6). This is possible due to the partial hypoellipticity of the operator $P$. 
As it was done in [37, it is convenient to get rid of the term $A\left(\mu \partial_{\mu}+\nu \partial_{\nu}\right)$ in (2.2), and to achieve this we conjugate the operator by $\theta^{-\frac{1}{4}}$. We get that

$$
\widetilde{Q}=\theta^{\frac{1}{4}} \widetilde{P} \theta^{-\frac{1}{4}}=\partial_{\mu} \partial_{\nu}-\mu \nu \Delta_{h}+\mu \nu B\left(\mu \nu, y, \partial_{y}\right)+C(\mu \nu, y)
$$

where $C$ is $C^{\infty}$ and $B\left(\mu \nu, y, \partial_{y}\right)=\sum_{j=1}^{N} b_{j}(\mu \nu, y) \partial_{y_{j}}$, with $b_{j} C^{\infty}$. Let $\widetilde{W}=\theta^{\frac{1}{4}} W$, then $\widetilde{Q} \widetilde{W}=0$. For $\phi(y) \in C_{0}^{\infty}(U)$, with $U \subset \subset \Gamma$, let

$$
G(\mu, \nu)=\int_{\partial X} \widetilde{W}(\mu, \nu, y) \phi(y) d y
$$

Let $Z\left(\mu, \nu, y, D_{y}\right)=\mu \nu \Delta_{h}-\mu \nu B\left(\mu \nu, y, \partial_{y}\right)-C(\mu \nu, y)$, and let $Z^{*}\left(\mu \nu, y, D_{y}\right)$ denote its transpose with respect to the $L^{2}(\partial X)$ product defined by (2.12), then

$$
\partial_{\mu} \partial_{\nu} G(\mu, \nu)=\int_{\partial X} \widetilde{W}(\mu, \nu, y) Z^{*}\left(\mu \nu, D_{y}\right) \phi(y) d y
$$

It follows from (2.9) that there exists $C>0$ such that

$$
\begin{aligned}
& \int_{0}^{T}\left|\partial_{\mu} \partial_{\nu} G(\mu, T)\right|^{2} d \mu \leq C\left(\sum_{|\alpha| \leq 2} \sup \left|\partial_{y}^{\alpha} \phi\right|\right)^{2}\|f\|_{L^{2}(X)}^{2}, \text { for } T \in(0, \varepsilon), \\
& \int_{\mu_{0}}^{T}\left|\partial_{\mu} \partial_{\nu} G\left(\mu_{0}, \nu\right)\right|^{2} d \nu \leq C\left(\sum_{|\alpha| \leq 2} \sup \left|\partial_{y}^{\alpha} \phi\right|\right)^{2}\|f\|_{L^{2}(X)}^{2}, \text { for } \mu_{0} \in(0, \varepsilon) .
\end{aligned}
$$

Let us denote $K=\left(\sum_{|\alpha| \leq 2} \sup \left|\partial_{y}^{\alpha} \phi\right|\right)\|f\|_{L^{2}(X)}$. Therefore, if $\delta<\mu<\varepsilon$,

$$
\left|\partial_{\nu} G(\mu, \nu)-\partial_{\nu} G(\delta, \nu)\right|=\left|\int_{\delta}^{\mu} \partial_{s} \partial_{\nu} G(s, \nu) d s\right| \leq C K(\mu-\delta)^{\frac{1}{2}} .
$$

Hence, for $\nu>0$,

$$
\limsup _{\delta \rightarrow 0}\left|\partial_{\nu} G(\delta, \nu)\right| \leq \liminf _{\mu \rightarrow 0}\left|\partial_{\nu} G(\mu, \nu)\right| .
$$

Hence, $\lim _{\mu \rightarrow 0}\left|\partial_{\nu} G(\mu, \nu)\right|$ exists. On the other hand, since $\mathcal{R}_{+}(0, f)(s, y)=0, y \in \Gamma$ and $s \leq s_{0}$, so, according to (2.8) it follows that

$$
\partial_{\nu} G(0, \nu)=0, \quad \nu \in\left(0, e^{\frac{s_{0}}{2}}\right) .
$$

Now we use (2.14) to show that if $\nu \in\left(0, e^{\frac{s_{0}}{2}}\right)$, then there exists $C>0$

$$
\left|\partial_{\nu} G(\mu, \nu)\right|=\left|\int_{0}^{\mu} \partial_{\mu} \partial_{\nu} G(\mu, \nu) d \mu\right| \leq C K \mu^{\frac{1}{2}}
$$

Since $W(\mu, \mu, y)=0$, we have for $\mu<\nu$,

$$
|G(\mu, \nu)|=\left|\int_{\mu}^{\nu} \partial_{s} G(\mu, s) d s\right| \leq C K \mu^{\frac{1}{2}}(\nu-\mu)^{\frac{1}{2}} .
$$

This shows that for every $\phi \in C_{0}^{\infty}(U)$

$$
\begin{gathered}
\left|\int_{\partial X} \widetilde{W}(\mu, \nu, y) \phi(y) d y\right| \leq C K \mu^{\frac{1}{2}} \\
\left|\int_{\partial X} \partial_{\nu} \widetilde{W}(\mu, \nu, y) \phi(y) d y\right| \leq C K \mu^{\frac{1}{2}} .
\end{gathered}
$$


Since $C_{0}^{\infty}\left(\mathbb{R}^{2}\right) \times C_{0}^{\infty}(U)$ is dense in $C_{0}^{\infty}\left(\mathbb{R}^{2} \times U\right)$, it follows that for any $\psi(\mu, \nu, y)$, with $|\mu| \leq \varepsilon$, $|\nu| \leq \varepsilon$,

$$
\begin{gathered}
\left|\int_{\partial X} \widetilde{W}(\mu, \nu, y) \psi(\mu, \nu, y) d y\right| \leq C\left(\sum_{|\alpha| \leq 2} \sup \left|\partial_{y}^{\alpha} \psi\right|\right)\|f\|_{L^{2}(X)} \mu^{\frac{1}{2}} \\
\left|\int_{\partial X} \partial_{\nu} \widetilde{W}(\mu, \nu, y) \psi(\mu, \nu, y) d y\right| \leq C\left(\sum_{|\alpha| \leq 2} \sup \left|\partial_{y}^{\alpha} \psi\right|\right)\|f\|_{L^{2}(X)} \mu^{\frac{1}{2}} .
\end{gathered}
$$

Now we differentiate (2.13) with respect to $\partial_{\nu}$. We have for $|\mu|<\varepsilon,|\nu|<\varepsilon$,

$$
\left.\partial_{\nu} \partial_{\mu} \partial_{\nu} G(\mu, \nu)=\int_{\partial X}\left[\partial_{\nu} \widetilde{W}(\mu, \nu, y) Z^{*}\left(\mu \nu, D_{y}\right) \phi(y)+\widetilde{W}(\mu, \nu, y) \partial_{\nu} Z^{*}(\mu \nu, y)\right) \phi(y)\right] d y,
$$

and so we obtain from (2.17)

$$
\left|\partial_{\mu} \partial_{\nu}^{2} G(\mu, \nu, y)\right| \leq C\left(\sum_{|\alpha| \leq 4}\left|\sup \partial_{y}^{\alpha} \phi\right|\right)|| f \|_{L^{2}(X)} \mu^{\frac{1}{2}}
$$

Let us denote $K_{N}(\psi)=\left(\sum_{|\alpha| \leq N}\left|\sup \partial_{y}^{\alpha} \phi\right|\right)\|f\|_{L^{2}(X)}$. Since $\widetilde{W}(\mu, \mu, y)=0$, it follows that $\partial_{\mu} \partial_{\nu} G(\mu, \mu)=0$, and so we have

$$
\left|\partial_{\mu} \partial_{\nu} G(\mu, \nu)\right|=\left|\int_{\mu}^{\nu} \partial_{\mu} \partial_{s}^{2} G(\mu, s) d s\right| \leq K_{4}(\phi) \mu^{\frac{1}{2}}
$$

On the other hand, since $W(\mu, \mu, y)=0$, it follows that $\left(\partial_{\mu} W\right)(\mu, \mu, y)=-\left(\partial_{\nu} W\right)(\mu, \mu, y)$. In particular, when $\nu=\mu$, we have

$$
\left|\partial_{\mu} G(\mu, \mu)\right| \leq C K_{2}(\phi) \mu^{\frac{1}{2}}
$$

and since

$$
\partial_{\mu} G(\mu, \nu)=\left(\partial_{\mu} G\right)(\mu, \mu)+\int_{\mu}^{\nu} \partial_{s} \partial_{\mu} G(\mu, s) d s
$$

we have

$$
\left|\partial_{\mu} G(\mu, \nu)\right| \leq C\left(K_{2}(\phi)+K_{4}(\phi)\right) \mu^{\frac{1}{2}} .
$$

Proceeding as above, since $\partial_{\nu} G(0, \nu)=0$, it follows from (2.18) that $\left|\partial_{\nu} G(\mu, \nu)\right| \leq C K_{4}(\phi) \mu^{\frac{3}{2}}$, and since $G(\mu, \mu)=0$, then $|G(\mu, \nu)| \leq C K_{4}(\phi) \mu^{\frac{3}{2}}$, and $\left|\partial_{\mu} \partial_{\nu}^{2} G(\mu, \nu)\right| \leq C K_{6}(\phi) \mu^{\frac{3}{2}}$. So iterating this argument, and using the symmetry of $W$ we get that for $k \geq 0$,

$$
\begin{aligned}
\partial_{\mu}^{k} G(0, \nu) & =0, \quad \partial_{\nu}^{k} G(\mu, 0)=0, \\
\left|\left(\partial_{\mu} G\right)(\mu, \mu)\right| & =\left|\left(\partial_{\nu} G\right)(\mu, \mu)\right| \leq C \mu^{k} .
\end{aligned}
$$

In particular this shows that, in the sense of distributions, $\widetilde{W}$ can be extended across the wedge $\{\mu=0\} \cup\{\nu=0\}$ by setting $\widetilde{W}(\mu, \nu, y)=0$ if $\mu, \nu \in(-\varepsilon, 0]$, satisfying

$$
\widetilde{Q} \widetilde{W}=0 \text { in }(-\varepsilon, \varepsilon) \times(-\varepsilon, \varepsilon) \times \Gamma .
$$

We also know more about the regularity of $\widetilde{W}$ in the variable $y$. From (2.9) we have

$$
\int_{-\varepsilon}^{\varepsilon} \int_{-\varepsilon}^{\varepsilon} \int_{\partial X}\left[|\widetilde{W}|^{2}+\mu\left|\partial_{\mu} \widetilde{W}\right|^{2}+\nu\left|\partial_{\nu} \widetilde{W}\right|^{2}+\mu \nu(\mu+\nu)\left|d_{h(\mu \nu)} \widetilde{W}\right|^{2}\right] d y d \mu d \nu \leq C\|f\|_{L^{2}(X)}
$$



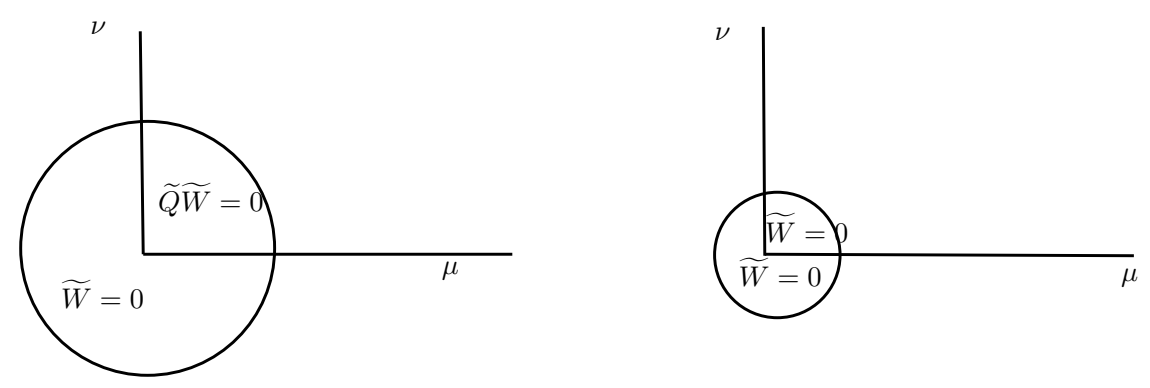

Figure 4 . If $\widetilde{Q} \widetilde{W}=0$ and $\widetilde{W}=0$ in the region on the left, then $\widetilde{W}=0$ in the region on the right.

The next step is to prove the following unique continuation result

Lemma 2.6. Let $\Gamma \subset \partial X$ be open and let $\widetilde{W}(\mu, \nu, y) \in C^{\infty}\left((-\varepsilon, \varepsilon) \times(-\varepsilon, \varepsilon) ; L^{2}(\Gamma)\right)$ satisfy (2.21) and be such that for $y \in \Gamma, \widetilde{W}(\mu, \nu, y)$ is supported in $\{\mu \geq 0, \nu \geq 0\}$. If

$$
\widetilde{Q} \widetilde{W}(\mu, \nu, y)=0, \text { in }(-\varepsilon, \varepsilon) \times(-\varepsilon, \varepsilon) \times \Gamma
$$

then for any $p \in \Gamma$ there exists $\delta>0$ and such that $W(\mu, \nu, y)=0$ provided $|\mu|<\delta,|\nu|<\delta$ and $|y-p|<\delta$.

Proof. As usual, the proof of this result is based on a Carleman estimate. First, it is convenient to make the change of variables

$$
r=\frac{\mu+\nu}{2}, \tau=\frac{\mu-\nu}{2}
$$

and we have

$$
\widetilde{Q}_{1}=4 \widetilde{Q}=\partial_{r}^{2}-\partial_{\tau}^{2}-4\left(r^{2}-\tau^{2}\right) \Delta_{h}+\left(r^{2}-\tau^{2}\right) B_{1}\left(r, \tau, y, D_{y}\right)+C_{1}(r, \tau, y) .
$$

Here, $h=h\left(r^{2}-\tau^{2}\right), B_{1}\left(r, \tau, y, D_{y}\right)=4 B\left(\left(r^{2}-\tau^{2}\right), y, D_{y}\right)$ and $C_{1}(r, \tau, y)=4 C\left(\left(r^{2}-\tau^{2}\right), y\right)$, and

$$
\widetilde{W}(r, \tau, y)=W(r+\tau, r-\tau, y), \quad \widetilde{W} \text { is supported in }|\tau| \leq r .
$$

Since the fibers over any fixed $(r, \tau)$ are not compact, one would have to cut-off in the variable $y$ to obtain the desired Carleman estimate. However this would produce error terms that could not be controlled. One needs to convexify the support of the solution $\widetilde{W}$. For small $\delta$, we choose local coordinates $y$ valid in $B(p, \delta)$ such that $p=0$, and set

$$
\rho=r+|y|^{2} \text {. }
$$

In this case the region which contains the support of $W,|\tau| \leq r$, can be described by

$$
|\tau|+|y|^{2} \leq \rho
$$

and the operator $\widetilde{Q}_{1}$ can be written as

$$
\widetilde{Q}_{1}=(1+F \varphi) \partial_{\rho}^{2}-\partial_{\tau}^{2}+4 \varphi \sum_{i j} h^{i j} \partial_{y_{i}} \partial_{y_{j}}+\sum_{j} \varphi R_{j} \partial_{y_{j}} \partial_{\rho}+\sum_{j} \varphi B_{j} \partial_{y_{j}}+H \varphi \partial_{\rho}+D,
$$

where $\varphi=\left(\rho-|y|^{2}\right)^{2}-\tau^{2}$ and $F=F(\rho, \tau, y), h^{i j}=h^{i j}(\rho, \tau, y), R_{j}=R_{j}(\rho, \tau, y), B_{j}=B_{j}(\rho, \tau, y)$, $D=D(\rho, \tau, y)$ and $H=H(\rho, \tau, y)$ are $C^{\infty}$ functions. Here we used that $\Delta_{h}$ is the positive 
Laplacian, see (2.3), and hence the sign of the third term. Moreover, there exists a constant $C$ such that for small $\varepsilon$,

$$
\sum_{i, j=1}^{n} h^{i j}(\rho, \tau, y) \xi_{i} \xi_{j} \geq C \sum_{j} \xi_{j}^{2}, \quad|(\rho, \tau)|<\varepsilon, \quad y \in \partial X .
$$

Let

$$
\begin{gathered}
\widetilde{Q}_{k}=\rho^{-k} \widetilde{Q}_{1} \rho^{k}=\widetilde{Q}+k \mathcal{L}+k(k-1) \rho^{-2}(1+\varphi F)-k \rho^{-1} \varphi H, \text { where } \mathcal{L} \text { is the vector field } \\
\mathcal{L}=2(1+\varphi F) \rho^{-1} \partial_{\rho}+\rho^{-1} \varphi \sum_{j} R_{j} \partial_{y_{j}}
\end{gathered}
$$

In what follows we will denote the inner product

$$
\begin{gathered}
\langle u, v\rangle=\int_{0}^{\varepsilon} \int_{0}^{\varepsilon} \int_{\partial X} u(\rho, \tau, y) \bar{v}(\rho, \tau, y) d y d \rho d \tau, \\
\text { and }\|u\|^{2}=\langle u, u\rangle
\end{gathered}
$$

for $\varepsilon$ small enough such that these coordinates are valid. We want to estimate the product

$$
\left\langle\widetilde{Q}_{k} V, \mathcal{L} V\right\rangle \text { for } V \in C_{0}^{\infty}((-\gamma, \gamma) \times(-\gamma, \gamma) \times \partial X) \text {, supported in }|\tau|+|y|^{2} \leq \rho .
$$

Without loss of generality, we assume that $V$ is real valued. From (2.23) we find that $\varphi \rho^{-2} \leq C$, on the support of $V$ and so, for $\gamma$ small enough, again using that $|\tau| \leq \rho-|y|^{2}$, there exists a constant $M>0$ such that first term of this product satisfies

$$
\begin{gathered}
\left\langle\left((1+\varphi F) \partial_{\rho}^{2}-\partial_{\tau}^{2}\right) V, \mathcal{L} V\right\rangle \geq \\
M\left(\left\|\rho^{-1} \partial_{\rho} V\right\|^{2}+\left\|\rho^{-1} \partial_{\tau} V\right\|^{2}-\sum_{j}\left\|\rho^{-\frac{1}{4}}\left(\rho-|y|^{2}\right)^{\frac{1}{2}} \partial_{y_{j}} V\right\|^{2}\right) .
\end{gathered}
$$

For $\gamma$ small enough, the second term can be bounded by

$$
\sum_{i j}\left\langle\varphi h^{i j} \partial_{y_{i}} \partial_{y_{j}} V, \mathcal{L} V\right\rangle \geq M\left(\sum_{j}\left\|\rho^{-\frac{1}{2}}\left(\rho-|y|^{2}\right)^{\frac{1}{2}} \partial_{y_{j}} V\right\|^{2}-\left\|\rho^{-\frac{1}{2}} \partial_{\rho} V\right\|^{2}\right) .
$$

For $\gamma$ small enough, we also have

$$
\sum_{j k}\left\langle\varphi R_{k} \partial_{y_{k}} \partial_{\rho} V, \mathcal{L} V\right\rangle \geq-M\left(\sum_{j}\left\|\left(\rho-|y|^{2}\right) \partial_{y_{j}} V\right\|^{2}-\left\|\partial_{\rho} V\right\|^{2}-\|V\|^{2}\right),
$$

and

$$
\left\langle\left(\varphi \sum_{j} B_{j} \partial_{y_{j}}+D+\varphi H \partial_{\rho}\right) V, \mathcal{L} V\right\rangle \geq-M\left(\|V\|^{2}+\left\|\rho^{-1} \partial_{\rho} V\right\|^{2}+\sum_{j}\left\|\left(\rho-|y|^{2}\right)^{\frac{1}{2}} \partial_{y_{j}} V\right\|^{2}\right) .
$$

Finally,

$$
\left\langle\left(k(k-1) \rho^{-2}(1+\varphi F)+k \rho^{-1} \varphi H\right) V, \mathcal{L} V\right\rangle \geq M k^{2}\left\|\rho^{-2} V\right\|^{2} .
$$

Putting together terms (2.25), (2.26), (2.27) (2.28) and (2.29) we deduce that, for $\gamma$ small enough, there exists $M>0$

$$
\left\langle\widetilde{Q}_{k} V, \mathcal{L} V\right\rangle \geq M\left(\left\|\rho^{-1} \partial_{\rho} V\right\|^{2}+\left\|\rho^{-1} \partial_{\tau} V\right\|^{2}+\sum_{j}\left\|\rho^{-\frac{1}{2}}\left(\rho-|y|^{2}\right) \partial_{y_{j}} V\right\|^{2}+k\|\mathcal{L} V\|^{2}+k^{2}\left\|\rho^{-2} V\right\|^{2}\right) .
$$

Since,

$$
\left\langle\widetilde{Q}_{k} V, \mathcal{L} V\right\rangle \leq \frac{1}{2}\left(\left\|\widetilde{Q}_{k} V\right\|^{2}+\|\mathcal{L} V\|^{2}\right)
$$


then, if $k$ is large enough and $\gamma$ is small enough,

$$
\left\|\widetilde{Q}_{k} V\right\|^{2} \geq M\left(\left\|\rho^{-1} \partial_{\rho} V\right\|^{2}+\left\|\rho^{-1} \partial_{\tau} V\right\|^{2}+\left\|\rho^{-\frac{1}{2}}\left(\rho-|y|^{2}\right)^{\frac{1}{2}} \nabla_{y} V\right\|^{2}+\frac{k}{2}\|\mathcal{L} V\|^{2}+k^{2}\left\|\rho^{-2} V\right\|^{2}\right) .
$$

Let $\chi \in C^{\infty}(\mathbb{R}), \chi(\rho)=1$ if $\rho \in\left(-\frac{\gamma}{2}, \frac{\gamma}{2}\right)$ and $\chi(\rho)=0$ if $|\rho|>\frac{3 \gamma}{4}$. Since $W$ is supported in $\mu \geq 0, \nu \geq 0$, it follows that in coordinates $(\rho, \tau, y), \widetilde{W}$ is supported in $|\tau|+|y|^{2} \leq \rho$, and hence $V=\chi(\rho) \widetilde{\widetilde{W}}$ is compactly supported for small $\gamma$. We would like to apply (2.30) to $V=\chi(\rho) \widetilde{W}$, but $\widetilde{W}$ is not necessarily smooth up to $\{\mu=0\},\{\nu=0\}$. So we have to molify $\widetilde{W}$ in the $y$-variable, and we let $\psi(y)$ be a $C_{0}^{\infty}$ function supported in $|y-p|<\delta$, with $\int \psi(y) d y=1$, and define $\psi_{m}(y)=m^{n} \psi(m y)$. Then for $m$ large,

$$
\widetilde{W}_{m}=\chi(\rho) \psi_{m} * \widetilde{W} \in C_{0}^{\infty}((-\varepsilon, \varepsilon) \times(-\varepsilon, \varepsilon) \times \Gamma),
$$

and since $\widetilde{Q}_{k}=\rho^{-k} \widetilde{Q}_{1} \rho^{k}$, we deduce from (2.30) that

$$
\begin{gathered}
\left\|\rho^{-k} \widetilde{Q} \widetilde{W}_{m}\right\|^{2} \geq \\
\left.M\left(k^{2}\left\|\rho^{-2-k} \widetilde{W}_{m}\right\|^{2}+\left\|\rho^{-\frac{1}{2}-k}\left(\rho-|y|^{2}\right)^{\frac{1}{2}} \nabla_{y} \widetilde{W}_{m}\right\|^{2}+\left\|\rho^{-1} \partial_{\rho}\left(\rho^{-k} \widetilde{W}_{m}\right)\right\|^{2}+\| \rho^{-1-k} \partial_{\tau} \widetilde{W}_{m}\right) \|^{2}\right) .
\end{gathered}
$$

To get an estimate for $\chi(\rho) \widetilde{W}$ from this one we use Friedrich's lemma to handle the commutators of $\widetilde{Q}$ and $\psi_{m}$. We use (2.21) and Theorem 2.4.3 of [18] to show that

$$
\begin{gathered}
\left\|\varphi \chi(\rho)\left(h^{i j} \partial_{y_{i}} \partial_{y_{j}}\left(\psi_{m} * \widetilde{W}\right)-\left(h^{i j} \partial_{y_{i}} \partial_{y_{j}} \widetilde{W}\right) * \psi_{m}\right)\right\|_{L^{2}} \leq C\left\|\chi(\rho) \varphi \nabla_{y} \widetilde{W}\right\|_{L^{2}}, \\
\left.\left\|\varphi h^{i j} \partial_{y_{i}}\left(\psi_{m} * \partial_{\rho}\left(\rho^{-k} \chi(\rho) \widetilde{W}\right)\right)-\varphi\left(h^{i j} \partial_{y_{i}} \partial_{\rho}\left(\rho^{-k} \chi(\rho) \widetilde{W}\right)\right) * \psi_{m}\right\|\right|_{L^{2}} \leq C\left\|\varphi \partial_{\rho} \rho^{-k} \chi(\rho) \widetilde{W}\right\|_{L^{2}}, \\
\left\|\rho^{-k} \chi(\rho)\left(\rho-|y|^{2}\right)^{\frac{1}{2}}\left(\nabla_{y}\left(\psi_{m} * \widetilde{W}\right)-\left(\nabla_{y} \widetilde{W}\right) * \psi_{m}\right)\right\|_{L^{2}} \leq C\left\|\rho^{-k} \chi(\rho)\left(\rho-|y|^{2}\right)^{\frac{1}{2}} \widetilde{W}\right\|_{L^{2}},
\end{gathered}
$$

and moreover

$$
\begin{gathered}
\lim _{m \rightarrow \infty}\left\|\varphi \chi(\rho)\left(h^{i j} \partial_{y_{i}} \partial_{y_{j}}\left(\psi_{m} * \widetilde{W}\right)-\left(h^{i j} \partial_{y_{i}} \partial_{y_{j}} \widetilde{W}\right) * \psi_{m}\right)\right\|_{L^{2}}=0 \\
\lim _{m \rightarrow \infty}\left\|\varphi h^{i j} \partial_{y_{i}}\left(\psi_{m} * \partial_{\rho} \rho^{-k} \chi(\rho) \widetilde{W}\right)-\varphi\left(h^{i j} \partial_{y_{i}} \partial_{\rho} \rho^{-k} \chi(\rho) \widetilde{W}\right) * \psi_{m}\right\|_{L^{2}}=0 \\
\lim _{m \rightarrow \infty}\left\|\rho^{-k} \chi(\rho)\left(D\left(\psi_{m} * \widetilde{W}\right)-(D \widetilde{W}) * \psi_{m}\right)\right\|_{L^{2}}=0 .
\end{gathered}
$$

Using these estimates, and letting $m \rightarrow \infty$ in (2.31) we obtain, for $k$ large enough,

$$
\begin{gathered}
\left\|\rho^{-k} \widetilde{Q}_{1} \chi(\rho) \widetilde{W}\right\|^{2} \geq \\
M\left(k^{2}\left\|\rho^{-2-k} \chi(\rho) \widetilde{W}\right\|^{2}+\left\|\rho^{-k-\frac{1}{2}}\left(\rho-|y|^{2}\right) \chi(\rho) \nabla_{y} \widetilde{W}\right\|^{2}+\left\|\rho^{-1} \partial_{\rho}\left(\rho^{-k} \widetilde{W}\right)\right\|^{2}+\| \rho^{-1-k} \partial_{\tau}\left(\chi(\rho) \widetilde{W} \|^{2}\right) .\right.
\end{gathered}
$$

Since $\widetilde{Q}_{1} \widetilde{W}=0$ and $\widetilde{Q}_{1}(\chi(\rho) \widetilde{W})=\left[\widetilde{Q}_{1}, \chi(\rho)\right] \widetilde{W}$ is supported in $\rho \geq \frac{\gamma}{2}$, we deduce from (2.32) that there exists $C=C(\widetilde{W})>0$ such that

$$
C\left(\frac{\gamma}{2}\right)^{-k} \geq M k^{2}\left\|\rho^{-2-k} \widetilde{W}\right\|^{2} \geq M k^{2}\left\|\rho^{-2-k} \widetilde{W}\right\|_{L^{2}\left(\rho \leq \frac{\gamma}{2}\right)}^{2} \geq M k^{2}\left(\frac{\gamma}{2}\right)^{-k-2}\|\widetilde{W}\|_{L^{2}\left(\rho \leq \frac{\gamma}{2}\right)}^{2}
$$


Hence

$$
k^{2}\|\widetilde{W}\|_{L^{2}\left(\rho \leq \frac{\gamma}{2}\right)}^{2} \leq C,
$$

and therefore $\widetilde{W}=0$ if $\rho \leq \frac{\gamma}{2}$. Returning to coordinates $(r, \tau, y)$, we obtain $\widetilde{W}=0$ if $r+|y|^{2} \leq \frac{\gamma}{2}$ and so $\widetilde{W}(r, \tau, y)=0$ i $r<\frac{\gamma}{4}$ and $|y| \leq \frac{\gamma}{4}$. This ends the proof of the Lemma.

Since $W(\mu, \nu, y)=V_{+}(\mu \nu, 2 \log \nu, y)$ satisfies the hypotheses of Lemma 2.6, we conclude that for any $p \in \Gamma$, there exists $\delta>0$ and such that

$$
V_{+}(\mu \nu, 2 \log \nu, y)=0 \text { provided }|y-p|<\delta \text {, and } \mu, \nu \in(-\delta, \delta) .
$$

In view of (2.5), we deduce that

$$
x^{-\frac{n}{2}} u(s-\log x, x, y)=V_{+}(x, s, y)=0 \text { provided }|y-p|<\delta, x \in(0, \delta) \text { and } \log x<s<\log \delta .
$$

We have also shown that $V_{+}$can be extended to the region $x<0$ such that, for $P$ as in (2.2)

$$
\begin{gathered}
P V_{+}=0 \\
V_{+}(x, s, y)=0 \quad x<0, \quad s<s_{0} \text { and } y \in \Gamma .
\end{gathered}
$$

The next step in the proof is

Proposition 2.7. Let $V(x, s, y)$ be in $H_{\mathrm{loc}}^{1}$ in the region $|x|<\varepsilon, y \in \partial X$ and $s \in \mathbb{R}$, satisfy $P V=0$, where $P$ is given by (2.2). Suppose $V(x, s, y)=0$ for $x \in(-\varepsilon, 0), s \leq s_{0}$ and $y \in \Gamma$. Let $s_{1}<s_{0}$ and $p \in \partial X$, and suppose that there exists $\delta>0$ such that $V(x, s, y)=0$ if $x<\delta$, $|y-p|<\delta$ and $s<s_{1}$. Then there exists $\beta \in(0, \delta)$ such that $V(x, s, y)=0$ if $x<\beta,|y-p|<\beta$ and $s<s_{1}+\frac{1}{4}\left(s_{0}-s_{1}\right)$. Figure 5 illustrates the result.
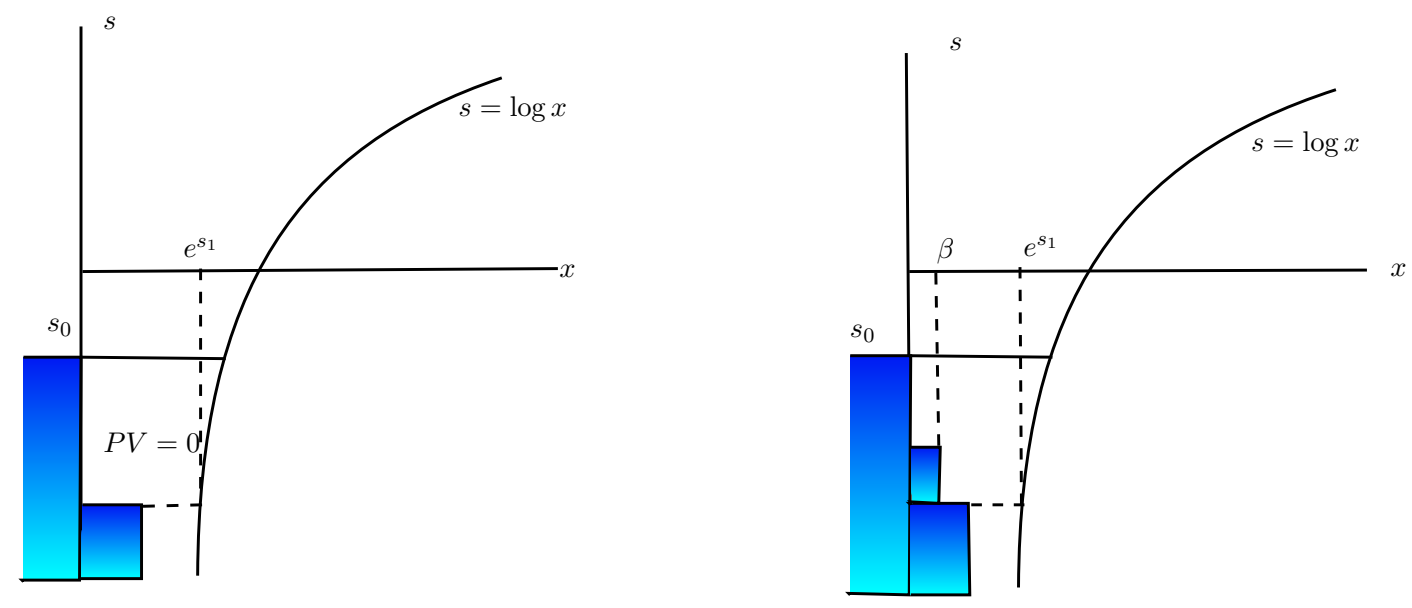

Figure 5. The unique continuation across the wedge $\left\{s<s_{1}, x<\delta,|y-p|<\right.$ $\delta\} \cup\left\{x<0, s<s_{0},|y-p|<\delta\right\}$. If $P V=0$ and $V=0$ in the colored region on the left, then $V=0$ in the colored region on the right. 
Proof. We will use unique continuation results due to Hörmander, and we will need to work with suitable strictly pseudoconvex surfaces. The key point here is that the operator $P$ is invariant under translations in the variable $s$. Let

$$
\varphi(x, s, y)=-x-k\left(s-s_{1}\right)-|y-p|^{2}, \text { where } k>0 \text { will be chosen later. }
$$

Since for $|y-p|<\delta, V=0$ if $x \in(-\varepsilon, 0]$ and $s<s_{0}$, or if $x<\delta$ and $x<s_{1}$, we have, see figure 5 ,

$$
V(x, s, y)=0 \text { if } \varphi>0,-\varepsilon<x<\delta, \quad \text { and }|y-p|<\delta .
$$

The principal symbol of the operator $P$ is

$$
p=-2 \sigma \xi-x \xi^{2}-x h(x, y, \eta),
$$

where $(\xi, \sigma, \eta)$ are the dual variables to $(x, s, y)$. Since $\nabla \varphi(x, s, y)=(-1,-k,-2(y-p))$, we have

$$
p(x, s, y, \nabla \varphi(x, s, y))=-2 k-x-h(x, y, 2(y-p)),
$$

$\varphi$ is not characteristic at $(x, s, y)$ if $x>-\frac{2 k}{1+h(x, y,-2(y-p))}>-k$ if $|y-p|<\beta$ is small enough

The Hamiltonian of $p$ is

$$
H_{p}=-2 \xi \partial_{s}-2(\sigma+x \xi) \partial_{x}-x H_{h}+\left(\xi^{2}+h+x \partial_{x} h\right) \partial_{\xi}
$$

where $H_{h}$ denotes the Hamiltonian of $h(x, y, \eta)$ in the variables $(y, \eta)$. Hence,

$$
\begin{gathered}
\left(H_{p} \varphi\right)(x, s, y, \xi, \sigma, \eta)=2(\sigma+x \xi)+2 k \xi+x H_{h}|y-p|^{2} \text { and } \\
\left(H_{p}^{2} \varphi\right)(x, s, y, \xi, \sigma, \eta)= \\
-2(\sigma+x \xi)\left(2 \xi+H_{h}|y-p|^{2}+x \partial_{x} H_{h}|y-p|^{2}\right)-\left(x H_{h}\right)^{2}|y-p|^{2}+2(k+x)\left(\xi^{2}+h+x \partial_{x} h\right) .
\end{gathered}
$$

If $H_{p} \varphi=0$, it follows that

$$
\begin{gathered}
H_{p}^{2} \varphi(x, s, y, \xi, \sigma, \eta)=2(x+3 k) \xi^{2}+2 \xi\left((k+x) H_{h}|y-p|^{2}+x \partial_{x} H_{h}|y-p|^{2}\right)+ \\
2(k+x)\left(h+x \partial_{x} h\right)+x\left(\left(H_{h}|y-p|^{2}\right)^{2}+x H_{h}|y-p|^{2} \partial_{x} H_{h}|y-p|^{2}-x H_{h}^{2}|y-p|^{2}\right) .
\end{gathered}
$$

If $|y-p|<\beta$ is small enough, there exists $C>0$ depending on $h$ only such that

$$
\left|H_{p}\right| y-\left.p\right|^{2}|\leq C \beta| \eta \mid, \text { and }\left|\partial_{x} H_{p}\right| y-\left.p\right|^{2}|\leq C \beta| \eta \mid \text {. }
$$

If we impose that $-\frac{k}{2}<x<\beta$, it follows that there exists $\varepsilon_{0}>0$ depending on $h$ such that if $\beta, k \in\left(0, \varepsilon_{0}\right)$ small, there exists $C>0$ such that

$$
h+x \partial_{x} h \geq C|\eta|^{2},
$$

and hence

$$
\begin{gathered}
H_{p}^{2} \varphi(x, s, p, \xi, \sigma, \eta) \geq k C\left(\xi^{2}-\beta|\xi||\eta|^{2}+|\eta|^{2}\right) \geq C k\left(\xi^{2}+|\eta|^{2}\right), \\
\text { if }-\frac{k}{2}<x<\beta \text { and } k, \delta \in\left(0, \varepsilon_{0}\right) .
\end{gathered}
$$

So we conclude that there exists $\varepsilon_{0}>0$ depending on $h$ such that

$$
\begin{gathered}
\text { if } p(x, s, y, \xi, \sigma, \eta)=H_{p} \varphi(x, s, y, \xi, \sigma, \eta)=0 \text { then } H_{p}^{2} \varphi(x, s, y, \xi, \sigma, \eta)>0 \\
\text { provided }(\xi, \sigma, \eta) \neq 0, \quad-\frac{k}{2}<x<\beta, \quad|y-p|<\beta, \quad k, \beta \in\left(0, \varepsilon_{0}\right) .
\end{gathered}
$$


Since $P$ is of second order, we deduce from (2.36) and (2.37) that the level surfaces of $\varphi$ are strictly pseudoconvex in the region

$$
-\frac{k}{2}<x<\beta, \quad|y-p|<\beta, \text { provided } k, \beta \in\left(0, \varepsilon_{0}\right) .
$$

As mentioned above, the invariance of $P$ under translations in $s$ imply that the conditions in (2.38) do not depend on $s$. Now we appeal to Theorem 28.2.3 and Proposition 28.3.3 of [19] and conclude that if

$$
Y=\left\{-\frac{k}{4}<x<\frac{\beta}{2},|y-p|<\frac{\beta}{\sqrt{2}},\left|s-s_{1}\right|<s_{0}-s_{1}\right\}
$$

there exist $C>0$ and $\lambda>0$ large such that if $\psi=e^{\lambda \varphi}$,

$$
C\left\|e^{\tau \psi} P v\right\|^{2} \geq \tau^{2}\left\|e^{\tau \psi} v\right\|^{2}+\tau\left\|e^{\tau \psi} v\right\|_{H^{1}}^{2} \text {, for all } v \in C_{0}^{\infty}(Y) \text { and } \tau \geq \tau_{0}>0 \text {. }
$$

Let $\theta \in C_{0}^{\infty}(Y)$ with $\theta=1$ if $-\frac{k}{8}<x<\frac{\beta}{4},|y-p|<\frac{\beta}{2}$ and $\left|s-s_{1}\right|<\alpha\left(s_{0}-s_{1}\right), \alpha<1$. Since $P V=0$, it follows that

$$
P(\theta V)=[P, \theta] V .
$$

But for $(x, s, y) \in Y, V(x, s, y)$ is supported in the region $x>0, s>s_{1}$, so we conclude that $P(\theta(x, s, y) V)$ is supported in $(x, s, y) \in Y \quad x \geq \frac{\beta}{4}, s-s_{1} \geq \alpha\left(s_{0}-s_{1}\right), \quad \alpha<1$ and $|y-p| \geq \frac{\beta}{2}$.

Therefore, by the definition of $\varphi$ we have

$$
\varphi(x, s, y) \leq-\min \left\{\frac{\beta}{4}, k \alpha\left(s_{0}-s_{1}\right), \frac{\beta^{2}}{4}\right\} \text { on the support of } P(\theta V) .
$$

Pick $k$ small so that $\min \left\{\frac{\beta}{4}, k \alpha\left(s_{0}-s_{1}\right), \frac{\beta^{2}}{4}\right\}=k \alpha\left(s_{0}-s_{1}\right)=\gamma$. Therefore we deduce from (2.39) and (2.40) that

$$
\tau^{2}\left\|e^{\tau\left(e^{\lambda \varphi}-e^{-\lambda \gamma}\right)} \theta V\right\|^{2} \leq C, \quad \tau>\tau_{0} .
$$

We remark that due to Friedrichs' Lemma, one can apply (2.39) to $\theta V$ even though it is not $C^{\infty}$, see [19]. Therefore, $\theta V=0$ if $e^{\lambda \varphi}-e^{-\lambda \gamma}>0$, so $\theta V=0$ if $\varphi>-\gamma$. So we deduce that

In particular,

$$
\theta V(x, s, y)=0 \text { provided } k\left(s-s_{1}\right)<\frac{\gamma}{3}, \quad 0<x<\frac{\gamma}{3}|y-p|^{2}<\frac{\gamma}{3} .
$$

$$
V(x, s, y)=0 \text { provided } s<s_{1}+\frac{\alpha}{3}\left(s_{0}-s_{1}\right), \quad \alpha<1, \quad 0<x<\frac{\gamma}{3}, \quad|y-p|^{2}<\frac{\gamma}{3} .
$$

This concludes the proof of Proposition 2.7.

The final ingredient in the proof of Theorem 2.2 is

Proposition 2.8. Let $u(t, z)$ satisfy (1.11) with initial data $f_{1}=0, f_{2}=f \in L_{a c}^{2}(X) \cap C^{\infty}(X)$. Let $V_{+}(x, s, y)=x^{-\frac{n}{2}} u(s-\log x, x, y)$. Let $p \in \partial X$, and suppose that there exist $s_{2} \in \mathbb{R}, \gamma>0$ and $\delta>0$ such that $V_{+}(x, s, y)=0$ if $0<x<\gamma, \log x<s<s_{2}$ and $|y-p|<\delta$. Then $u(t, z)=0$ for every $z \in X$ such that there exist $(x, y)$ with $x<\gamma$ and $|y-p|<\delta$ and $|t|+d_{g}(z ;(x, y)) \leq \log \left(\frac{e^{s_{0}}}{x}\right)$. In particular, if $s^{*}<s_{2}$ is such that coordinates $(x, y)$ for which (1.3) holds for $x<e^{s^{*}}$, then

$$
V_{+}(x, s, y)=0 \text { if }|y-p|<\delta, \quad 0<x<e^{s^{*}}, \text { and } \log x<s<s_{2} .
$$

Figure [6] illustrates the result. 


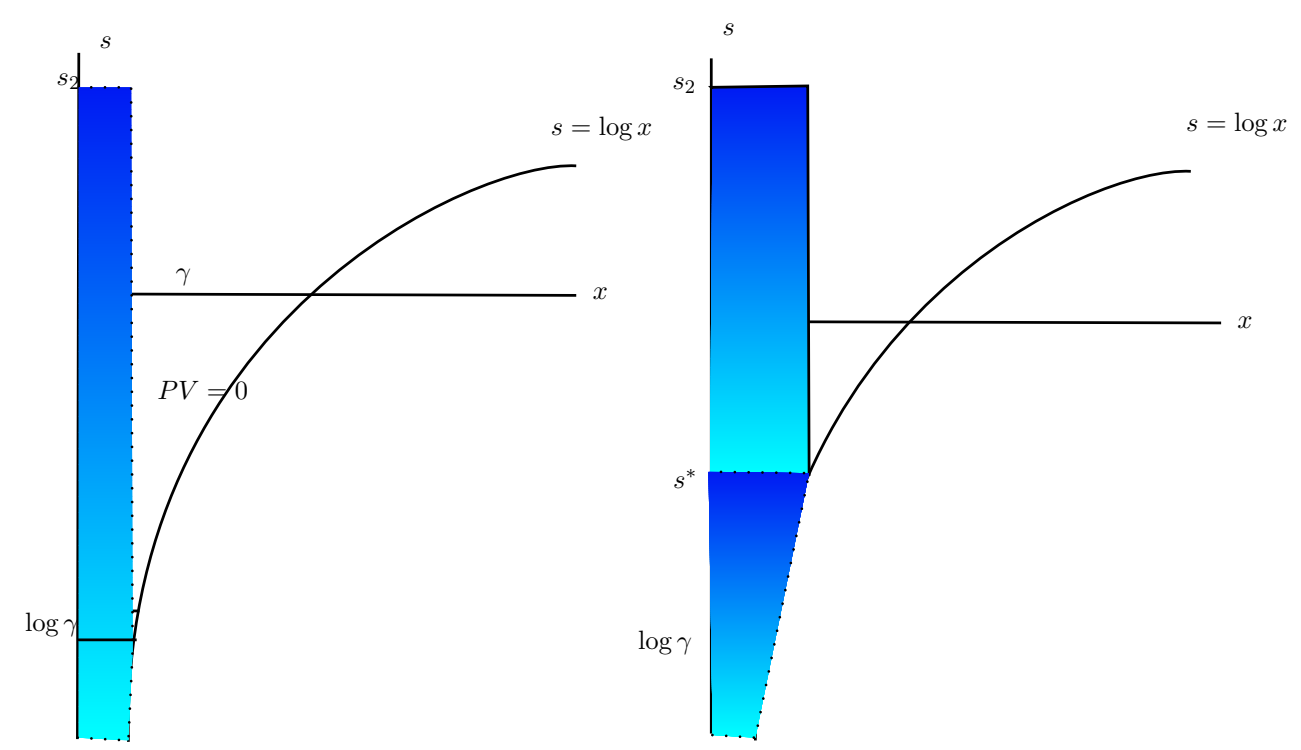

Figure 6 . If $P V=0$ and $V=0$ in the colored region on the left, then $V=0$ in the colored region on the right.

Proof. The key point in the proof is the following consequence of Tataru's theorem [39], see also [40, 17, 36]. If $u(t, z)$ is a $C^{\infty}$ function that satisfies

$$
\begin{gathered}
\left(D_{t}^{2}-\Delta_{g}+L\left(z, D_{z}\right)\right) u=0 \text { in }(\widetilde{T}, \widetilde{T}) \times \Omega, \\
u(t, z)=0 \text { in a neighborhood of }\left\{z_{0}\right\} \times(-T, T), \quad T<\widetilde{T},
\end{gathered}
$$

where $\Omega \subset \mathbb{R}^{n}, g$ is a $C^{\infty}$ Riemannian metric and $L$ is a first order $C^{\infty}$ operator (that does not depend on $t$ ), then

$$
u(t, z)=0 \text { if }|t|+d_{g}\left(z, z_{0}\right)<T,
$$

where $d_{g}$ is the distance measured with respect to the metric $g$.

Since the initial data of (1.11) is $(0, f), u(t, z)=-u(-t, z)$. If $x<\gamma, \log x<s<s_{1}$, and $|y-p|<\delta$, it follows from the definition of $V_{+}$that

$$
u(t, x, y)=0 \text { if } 0<x<\gamma, \quad|y-p|<\delta \text { and }|t| \leq s_{2}-\log x=\log \left(\frac{e^{s_{2}}}{x}\right) .
$$

Applying (2.43) with $z_{0}=(x, y)$ we obtain

$$
u(t, z)=0 \text { provided }|t|+d_{g}(z ;(x, y))<\log \left(\frac{e^{s_{2}}}{x}\right), \quad \text { with } 0<x<\delta,|y-p|<\delta .
$$

If $z=(\alpha, y)$ with $e^{s^{*}}>\alpha>x, d_{g}((x, y) ;(\alpha, y))=\log \left(\frac{\alpha}{x}\right)$, it follows from (2.43)

$$
u(t,(\alpha, y))=0 \text { if } t+\log \left(\frac{\alpha}{x}\right)<\log \left(\frac{e^{s 2}}{x}\right) .
$$

In particular this guarantees that $u(t, \alpha, y)=0$ if $0<t<\log \left(\frac{e^{s_{2}}}{\alpha}\right)$, and since $s=t+\log \alpha$, hence $V_{+}(\alpha, s, y)=0$ if $\alpha<e^{s^{*}}, s<s_{2}$ and $|y-p|<\delta$. This ends the proof of Proposition 2.8,

We can now conclude the proof of Theorem 2.2. 
Proof. We know from Proposition 2.5 that for any $p \in \Gamma$ there exists $\delta>0$ such that

$$
V_{+}(x, s, y)=0 \text { if } x<\delta, s<\log \delta,|y-p|<\delta .
$$

Moreover, $V(x, s, y)=0$ if $x<0, s<s_{0}$ and $y \in \Gamma$. Applying Proposition 2.7 with $s_{1}=\log \delta$, we find that there exists $\beta_{1}<\delta$ such that

$$
V_{+}(x, s, y)=0 \text { provided } x<\beta_{1}, \quad|y-p|<\beta_{1} \text { and } \log x<s<\log \delta+\frac{1}{4}\left(s_{0}-\log \delta\right) .
$$

Then Proposition 2.8 guarantees that there exists $s^{*}<<0$ such that,

$$
V_{+}(x, s, y)=0 \text { if } x<e^{s^{*}}, \quad|y-p|<\beta_{1}, \quad s<s_{2}=\log \delta+\frac{1}{4}\left(s_{0}-\log \delta\right) .
$$

Again using Proposition 2.7 and Proposition $2.8 n$ times, we find that there exists $\beta_{n}<\beta_{n-1}$ such that for $s_{n}=s_{n-1}+\frac{1}{3}\left(s_{0}-s_{n-1}\right)$,

$$
V_{+}(x, s, y)=0 \text { if } x<e^{s^{*}},|y-p|<\beta_{n-1}, \quad s_{n}=s_{n-1}+\frac{1}{4}\left(s_{0}-s_{n-1}\right) .
$$

The main point is that while the neighborhood of $p$ shrinks from one step to the next, the neighborhood of $x=0$ stays the same: $x<e^{s^{*}}$. Since $p$ is arbitrary, it follows that, for every $p \in \Gamma$, $V_{+}(x, s, p)=0$ provided $0<x<e^{s^{*}}$ and $\log x<s<s_{0}$.

We again resort to the consequence of Tataru's theorem to finish the proof of the result. Let $w=(\alpha, p)$, with $0<\alpha<e^{s^{*}}$ and $p \in \Gamma$, then the solution $u(t, z)$ vanishes in a neighborhood of $\{w\} \times\left(-\log \left(\frac{e^{s_{0}}}{\alpha}\right), \log \left(\frac{e^{s_{0}}}{\alpha}\right)\right)$. Therefore, by (2.43),

$$
u(t, z)=\partial_{t} u(t, z)=0 \text { if }|t|+d_{g}(z, w)<\log \left(\frac{e^{s_{0}}}{\alpha}\right) .
$$

In particular, when $t=0$ we find that $\partial_{t} u(0, z)=f(z)=0$ provided $d_{g}(z, w)<\log \left(\frac{e^{s_{0}}}{\alpha}\right)$, and this concludes the proof of Theorem 2.2.

The following result will be useful in the next section.

Corollary 2.9. Let $\Gamma \subset \partial X$ be open. If $f \in L_{a c}^{2}(X)$ and $\mathcal{R}_{+}(0, f)(s, y)=0$ for every $s \in \mathbb{R}$ and $y \in \Gamma$, then $f=0$. Similarly, if $(h, 0) \in E_{a c}(X)$ and $\mathcal{R}_{+}(h, 0)(s, y)=0$ for every $s \in \mathbb{R}$ and $y \in \Gamma$, then $h=0$.

Proof. If $\mathcal{R}(0, f)(s, y)=0$ for every $s \in \mathbb{R}$ and $y \in \Gamma$, then $f(z)=0$ if $z \in \mathcal{D}_{s_{0}}(\Gamma)$ for every $s_{0}$. Since $X$ is connected, the distance between any two points in the interior of $X$ is finite. Therefore $f=0$.

Suppose $F=\mathcal{R}(h, 0)(s, y)=0$ for every $s \in \mathbb{R}$ and $y \in \Gamma$. As in the proof of Theorem 2.2, by taking convolution of $F$ with $\phi \in C_{0}^{\infty}(\mathbb{R})$, we may assume that $\left(\Delta_{g}-\frac{n^{2}}{4}\right)^{k} h \in L_{a c}^{2}(X)$ for every $k \geq 0$. Let $u(t, z)$ satisfy (1.11) with initial data $(h, 0)$ and let $V=\partial_{t} u$. Then $V$ satisfies (1.11) with initial data $\left(0,\left(\Delta_{g}-\frac{n^{2}}{4}\right) h\right)$ and $\mathcal{R}_{+}\left(0,\left(\Delta_{g}-\frac{n^{2}}{4}\right) h\right)(s, y)=0$ for $s \in \mathbb{R}$ and $y \in \Gamma$. But as we have shown, this implies that $\left(\Delta_{g}-\frac{n^{2}}{4}\right) h=0$. Since $(h, 0) \in E_{a c}(X)$, this implies that $h=0$.

One should remark that this result can be proved by applying a result of Mazzeo [33], see also [42]. The solution to (1.11) with initial data $(0, f)$ is odd, and since $\mathcal{R}_{+}(0, f)(s, y)=0$, for $s \in \mathbb{R}, y \in \Gamma$, it follows that $\mathcal{R}_{-}(0, f)(s, y)=0$ for $s \in \mathbb{R}, y \in \Gamma$. Taking Fourier transform in $s$ we find that

$$
\left(\Delta_{g}-\lambda^{2}-\frac{n^{2}}{4}\right) \widehat{u}(\lambda, z)=0
$$


and using that $\mathcal{R}_{+}(0, f)(s, y)=\mathcal{R}_{-}(0, f)(s, y)=0$, one deduces by using a formal power series argument as in the proof of Proposition 3.4 of [12], that $\widehat{u}(\lambda, z)$ vanishes to infinite order at $\Gamma$. Theorem 14 of [33] implies that $\widehat{u}=0$ and hence $u=0$. In particular $f=0$.

\section{The Control Space}

One of the key arguments used in [37] to prove Theorem 1.2 was that the ranges of the forward and backward radiation fields

$$
\mathcal{M}^{ \pm}=\mathcal{R}_{ \pm}\left(0, L_{a c}^{2}(X)\right)=\left\{\mathcal{R}_{ \pm}(0, f): f \in L_{a c}^{2}(X)\right\}
$$

are closed subspaces of $L^{2}(\mathbb{R} \times \partial X)$, and are characterized by the scattering operator. The main goal of this section is to define an analogue of $\mathcal{M}^{ \pm}$for functions supported in $\mathbb{R} \times \Gamma$. Throughout the remaining of this paper we will denote

$$
L^{2}(\mathbb{R} \times \Gamma)=\left\{\left.F\right|_{\mathbb{R} \times \Gamma}: F \in L^{2}(\mathbb{R} \times \partial X)\right\},
$$

and $\left.\mathcal{S}\right|_{\mathbb{R} \times \Gamma}$ will denote the operator defined by

$$
\begin{gathered}
\left.\mathcal{S}\right|_{\mathbb{R} \times \Gamma}: L^{2}(\mathbb{R} \times \Gamma) \longrightarrow L^{2}(\mathbb{R} \times \Gamma) \\
\left.F \longmapsto(\mathcal{S} F)\right|_{\mathbb{R} \times \Gamma} .
\end{gathered}
$$

Since we assume we know $\left.\mathcal{A}(\lambda)\right|_{\Gamma}$ for every $\lambda$, then in view of (1.19) we may assume we know $\left.\mathcal{S}\right|_{\mathbb{R} \times \Gamma}$. We shall prove

Theorem 3.1. Let $\Gamma \subset \partial X$ be an open subset such that $\partial X \backslash \Gamma$ does not have empty interior. The space

$$
\mathcal{M}(\Gamma)^{ \pm}=\left\{\left.\mathcal{R}_{ \pm}(0, f)\right|_{\mathbb{R} \times \Gamma}: f \in L_{a c}^{2}(X)\right\},
$$

equipped with norm $\mathcal{N}$ defined by

$$
\mathcal{N}\left(\left.\mathcal{R}_{ \pm}(0, f)\right|_{\mathbb{R} \times \Gamma}\right)=\|f\|_{L^{2}(X)} .
$$

is a Hilbert space determined by $\left.\mathcal{S}\right|_{\mathbb{R} \times \Gamma}$.

Proof. We shall work with the forward radiation field. The proof of the result for $\mathcal{R}_{-}$is identical. Since $\mathcal{R}_{+}$is linear, the triangle inequality for the $L^{2}(X)$-norm implies that $\mathcal{N}$ is a norm, and that

$$
\left\langle\left.\mathcal{R}_{+}(0, f)\right|_{\mathbb{R} \times \Gamma},\left.\mathcal{R}_{+}(0, h)\right|_{\mathbb{R} \times \Gamma}\right\rangle_{\mathcal{N}}=\langle f, h\rangle_{L^{2}(X)}
$$

is an inner product. Since $\mathcal{R}_{+}$is continuous and $L_{a c}^{2}(X)$ is complete, it follows that $(\mathcal{M}(\Gamma), \mathcal{N})$ is a Hilbert space. We need to show that it is determined by $\left.\mathcal{S}\right|_{\mathbb{R} \times \Gamma}$, and we begin by observing, as in [37, that the symmetry of the wave equation under time reversal gives that

$$
\mathcal{R}_{+}\left(f_{1}, f_{2}\right)(-s, y)=\mathcal{R}_{+}\left(f_{1}, 0\right)(-s, y)+\mathcal{R}_{+}\left(0, f_{2}\right)(-s, y)=-\mathcal{R}_{-}\left(f_{1}, 0\right)(s, y)+\mathcal{R}_{-}\left(0, f_{2}\right)(s, y) .
$$

In particular, if $F(s, y)=\mathcal{R}_{+}\left(f_{1}, f_{2}\right)(s, y)$, and if we denote $F^{*}(s, y)=F(-s, y)$, then applying $\mathcal{S}$ to the second equality of (3.3) , we obtain

$$
\mathcal{S} F^{*}(s, y)=-\mathcal{R}_{+}\left(f_{1}, 0\right)+\mathcal{R}_{+}\left(0, f_{2}\right)=\mathcal{R}_{+}\left(-f_{1}, f_{2}\right),
$$

and hence we deduce that

$$
\text { if } F(s, y)=\mathcal{R}_{+}\left(f_{1}, f_{2}\right) \text {, then } \frac{1}{2}\left(F+\mathcal{S} F^{*}\right)=\mathcal{R}_{+}\left(0, f_{2}\right) \text {, and } \frac{1}{2}\left(F-\mathcal{S} F^{*}\right)=\mathcal{R}_{+}\left(f_{1}, 0\right),
$$


and therefore,

$$
\begin{gathered}
F(s, y)=\mathcal{R}_{+}\left(0, f_{2}\right), \text { if and only } F=\mathcal{S} F^{*}, \\
F(s, y)=\mathcal{R}_{+}\left(f_{1}, 0\right), \text { if and only if } F=-\mathcal{S} F^{*} .
\end{gathered}
$$

Since $\mathcal{R}_{+}$is an isometry, then for any $F \in L^{2}(\mathbb{R} \times \Gamma)$ there exists $(f, h) \in E_{a c}(X)$ such that $\mathcal{R}(f, h)=F$. But in view of the first identity in (3.5) we have

$$
\left.\frac{1}{2}\left(F+\mathcal{S} F^{*}\right)\right|_{\mathbb{R} \times \Gamma}=\left.\mathcal{R}_{+}(0, h)\right|_{\mathbb{R} \times \Gamma} .
$$

We shall denote

$$
\begin{gathered}
\mathcal{L}: L^{2}(\mathbb{R} \times \Gamma) \longrightarrow L^{2}(\mathbb{R} \times \Gamma) \\
\left.F \longmapsto \frac{1}{2}\left(F+\mathcal{S} F^{*}\right)\right|_{\mathbb{R} \times \Gamma}
\end{gathered}
$$

Since $\mathcal{S}$ is unitary, it follows that $\|\mathcal{L}\| \leq 1$. In fact, the map $\mathcal{L}$ gives more information:

Lemma 3.2. If $F=\mathcal{R}_{+}(f, h)$ is supported in $\mathbb{R} \times \bar{\Gamma}$, then $\|h\|_{L^{2}(X)}$ is determined by $\left.\mathcal{S}\right|_{\mathbb{R} \times \Gamma}$.

Proof. If $F(s, y)=\mathcal{R}_{+}(f, h) \in L^{2}(\mathbb{R} \times \Gamma)$, since $\mathcal{R}_{+}$is unitary, then according to (3.5)

$$
\begin{gathered}
\left\langle F,\left(F+\left.\left(\mathcal{S} F^{*}\right)\right|_{\mathbb{R} \times \Gamma}\right)\right\rangle=\left\langle F,\left(F+\mathcal{S} F^{*}\right)\right\rangle=\left\langle\mathcal{R}_{+}(f, h), \mathcal{R}_{+}(-f, h)\right\rangle= \\
\|h\|_{L^{2}(X)}^{2}-\left(\left\|d_{g} f\right\|_{L^{2}(X)}^{2}-\frac{n^{2}}{4}\|f\|_{L^{2}(X)}^{2}\right)
\end{gathered}
$$

On the other hand, $\|F\|_{L^{2}(\mathbb{R} \times \Gamma)}=\|h\|_{L^{2}(X)}^{2}+\left(\left\|d_{g} f\right\|_{L^{2}(X)}^{2}-\frac{n^{2}}{4}\|f\|_{L^{2}(X)}^{2}\right)$, therefore

$$
\frac{1}{2}\|F\|^{2}+\frac{1}{2}\left\langle F,\left(F+\left.\left(\mathcal{S} F^{*}\right)\right|_{\mathbb{R} \times \Gamma}\right)\right\rangle=\|h\|^{2} .
$$

We know from (1.16) that given $F \in L^{2}(\mathbb{R} \times \Gamma)$ there exists $(f, h) \in E_{a c}(X)$ such that $\mathcal{R}_{+}(f, h)=$ $F$. We can say the following about such initial data

Proposition 3.3. Let $\Gamma \subset \partial X$ be an open subset such that $\partial X \backslash \Gamma$ contains an open set $\mathcal{O}$, and $h \in L_{a c}^{2}(X)$. Then there exists at most one $f$ such that $(f, 0) \in E_{a c}(X)$ and $\mathcal{R}_{+}(f, h)$ is supported in $\mathbb{R} \times \Gamma$. Moreover, the set

$$
\mathcal{C}(\Gamma)=\left\{h \in L_{a c}^{2}(X): \text { there exists }(f, 0) \in E_{a c}(X) \text { such that } \mathcal{R}_{+}(f, h)(s, y)=0, y \in \partial X \backslash \Gamma\right\}
$$

is dense in $L_{a c}^{2}(X)$.

Proof. First, if $\mathcal{R}_{+}\left(f_{1}, h\right)$ and $\mathcal{R}_{+}\left(f_{2}, h\right)$ are supported in $\mathbb{R} \times \bar{\Gamma}$, then $\mathcal{R}_{+}\left(f_{1}-f_{2}, 0\right)$ is supported in $\mathbb{R} \times \bar{\Gamma}$, but this implies that $\mathcal{R}_{+}\left(f_{1}-f_{2}, 0\right)=0$ in $\mathbb{R} \times \mathcal{O}$, and so Corollarry 2.9 implies that $f_{1}=f_{2}$.

Let $v \in L_{a c}^{2}(X)$ and assume that $\langle v, h\rangle_{L^{2}(X)}=0$ for all $h \in \mathcal{C}(\Gamma)$. Since $\mathcal{R}_{+}$is unitary, then for all $(f, 0) \in E_{a c}(X)$,

$$
\langle v, h\rangle_{L^{2}(X)}=\left\langle\mathcal{R}_{+}(0, v), \mathcal{R}_{+}(f, h)\right\rangle_{L^{2}(\mathbb{R} \times \partial X)}
$$

Since $h \in \mathfrak{C}(\Gamma)$, is arbitrary, it follows that

$$
\left\langle\mathcal{R}_{+}(0, v), F\right\rangle_{L^{2}(\mathbb{R} \times \partial X)}=0 \text { for all } F \in L^{2}(\mathbb{R} \times \Gamma) .
$$

Hence $\mathcal{R}_{+}(0, v)=0$ on $\mathbb{R} \times \Gamma$ and by Corollary 2.9 , $v=0$. 
Lemma 3.4. If $\Gamma \subset \partial X$ is open and $\partial X \backslash \Gamma$ contains an open subset, then the map $\mathcal{L}$ is injective and has dense range.

Proof. If $F=\mathcal{R}_{+}(f, h)$, is supported in $\mathbb{R} \times \bar{\Gamma}$, then $\mathcal{L} F=\left.\mathcal{R}_{+}(0, h)\right|_{\mathbb{R} \times \Gamma}$. If $\mathcal{L} F=0$ then $\mathcal{R}_{+}(0, h)=$ 0 on $\mathbb{R} \times \Gamma$. It follows from Corollary 2.9 that $h=0$, and hence $F=\mathcal{R}(f, 0)$. Since there exists an open subset $\mathcal{O} \subset(\partial X \backslash \Gamma)$, and $F$ is supported in $\mathbb{R} \times \bar{\Gamma}$, it follows that $F=\mathcal{R}_{+}(f, 0)=0$ in $\mathbb{R} \times \mathcal{O}$, and again by Corollary $2.9, f=0$ and so $F=0$.

Now we prove that its range is dense. Let $H \in L^{2}(\mathbb{R} \times \Gamma)$ be orthogonal to the range of $\mathcal{L}$. In this case, since $H$ is supported in $\mathbb{R} \times \bar{\Gamma}$, then for every $F \in L^{2}(\mathbb{R} \times \Gamma)$,

$$
0=\left\langle H,\left.\left(F+\mathcal{S} F^{*}\right)\right|_{\mathbb{R} \times \Gamma}\right\rangle_{L^{2}(\mathbb{R} \times \Gamma)}=\left\langle H, F+\mathcal{S} F^{*}\right\rangle_{L^{2}(\mathbb{R} \times \Gamma)} .
$$

Since $\mathcal{S}$ is unitary its adjoint is its inverse $\mathcal{S}^{-1}$. Hence

$$
\begin{gathered}
0=\left\langle H, F+\mathcal{S} F^{*}\right\rangle_{L^{2}(\mathbb{R} \times \Gamma)}=\langle H, F\rangle_{L^{2}(\mathbb{R} \times \Gamma)}+\left\langle\mathcal{S}^{-1} H, F^{*}\right\rangle_{L^{2}(\mathbb{R} \times \Gamma)}= \\
\langle H, F\rangle+\left\langle\left(\mathcal{S}^{-1} H\right)^{*}, F\right\rangle_{L^{2}(\mathbb{R} \times \Gamma)}=\left\langle H+\left(\mathcal{S}^{-1} H\right)^{*}, F\right\rangle_{L^{2}(\mathbb{R} \times \Gamma)}
\end{gathered}
$$

Therefore $H+\left.\left(\mathcal{S}^{-1} H\right)^{*}\right|_{\mathbb{R} \times \Gamma}=0$. This implies that $H^{*}+\left.\left(\mathcal{S}^{-1} H\right)\right|_{\mathbb{R} \times \Gamma}=0$. If $H=\mathcal{R}_{+}\left(f_{1}, f_{2}\right)$, then by arguing as above, we see that $H^{*}+\mathcal{S}^{-1} H=2 \mathcal{R}_{-}\left(0, f_{2}\right)$. By Corollary 2.9, $f_{2}=0$. But since $H$ is supported in $\Gamma$ and $\partial X \backslash \Gamma$ contains an open subset, we deduce again from Corollary 2.9 that $f_{1}=0$, and thus $H=0$.

We shall denote

$$
\mathcal{F}(\Gamma)=\mathcal{L}\left(L^{2}(\mathbb{R} \times \Gamma)\right)=\left\{\left.\mathcal{R}_{+}(0, f)\right|_{\mathbb{R} \times \Gamma}: f \in \mathcal{C}(\Gamma)\right\},
$$

and equip $\mathcal{F}(\Gamma)$ with the norm given by Lemma 3.2

$$
\mathcal{N}\left(\mathcal{R}_{+}(0, f)\right)=\|f\|_{L^{2}(X)} .
$$

$(\mathcal{F}(\Gamma), \mathcal{N})$ is a normed vector space, and since $\mathcal{C}(\Gamma)$ is dense in $L^{2}(X), \mathcal{F}(\Gamma)$ is dense in $(\mathcal{M}(\Gamma), \mathcal{N})$. Hence $(\mathcal{M}(\Gamma), \mathcal{N})$ is the completion of $(\mathcal{F}(\Gamma), \mathcal{N})$ into a Hilbert space, and therefore it is determined by $\left.\mathcal{S}\right|_{\mathbb{R} \times \Gamma}$. Notice that the completion of $\mathcal{F}(\Gamma)$ with the $L^{2}(\mathbb{R} \times \Gamma)$-norm is $L^{2}(\mathbb{R} \times \Gamma)$. But

$$
\left\|\left.\left.\mathcal{R}_{+}(0, h)\right|_{(\mathbb{R} \times \Gamma)}\right|_{L^{2}(\mathbb{R} \times \Gamma)} \leq\right\| h \|_{L^{2}(X)}
$$

and hence, $\mathcal{N}$ is a stronger norm and $(\mathcal{M}(\Gamma), \mathcal{N})$ is a smaller space. This ends the proof of Theorem 3.1 .

\section{Proof of Theorem 1.3}

As in [37 and [7, first we construct an isometry between neighborhoods of $\Gamma$ which realizes (1.10). Then we apply the result of Kurylev and Lassas [26] on the reconstruction of a Riemannian manifold from the Dirichlet-to-Neumann on part of the boundary to show that the diffeomorphism between neighborhoods of $\Gamma$ can be extended to an isometry between the two manifolds.

First recall that the maps given by (1.3)

$$
\begin{gathered}
\Psi_{j, \varepsilon}:[0, \varepsilon) \times \partial X_{j} \longrightarrow U_{\varepsilon, j} \subset X_{j} \\
\left(x_{j}, y\right) \mapsto z
\end{gathered}
$$

put the metrics $g_{j}$ in the form

$$
\Psi_{j}^{*} g_{j}=\frac{d x_{j}^{2}}{x_{j}^{2}}+\frac{h_{j}\left(x_{j}, y, d y\right)}{x_{j}^{2}}
$$


As shown in [11, and discussed in section 1, $\Psi_{j, \varepsilon}\left(x_{j}, y\right)$ is the point obtained by flowing the integral curve of $\nabla_{x_{j}^{2} g_{j}} x_{j}$ emanating from the point $y \in \partial X_{j}$ by $x_{j}$ units of time. So $x_{j}$ is the arc-length along the geodesics of $x_{j}^{2} g_{j}$ normal to $\partial X_{j}$. We can pick $\varepsilon$ small such that both maps are diffeomorphisms. For $z=(x, y) \in \Gamma \times[0, \varepsilon), x(z)$ is the distance from $z$ to $\Gamma$ with respect to the metric $x_{j}^{2} g_{j}$. In particular, when $y \in \Gamma$,

$$
\Psi_{j}^{*} g_{j}=\frac{d x^{2}}{x^{2}}+\frac{h_{j}(x, y, d y)}{x^{2}}, y \in \Gamma
$$

Our first result will be

Proposition 4.1. Let $\left(X_{1}, g_{1}\right),\left(X_{2}, g_{2}\right)$ and $\Gamma$ satisfy the hypotheses of Theorem (1.3). Let $\mathcal{R}_{j, \pm}\left(s, y, x^{\prime}, y^{\prime}\right)$ denote the kernels of the radiation fields acting on pairs of the form $(0, f), f \in$ $L_{a c}^{2}(X)$. Then there exists $\epsilon>0$ such that in the product decomposition $[0, \epsilon) \times \Gamma$ where (4.1) holds, $h_{1}(x, y, d y)=h_{2}(x, y, d y)$ and

$$
\mathcal{R}_{1, \pm}\left(s, y, x^{\prime}, y^{\prime}\right)=\mathcal{R}_{2, \pm}\left(s, y, x^{\prime}, y^{\prime}\right), \text { if } y, y^{\prime} \in \Gamma, \quad x^{\prime}<\varepsilon .
$$

Proof. The proof of Proposition 4.1 is an adaptation of the Boundary Control Method to this setting. As in [37], pick $x_{1}<\varepsilon$, and consider the spaces

$$
\begin{gathered}
\mathcal{M}_{x_{1}}^{+}(\Gamma)=\left\{F \in \mathcal{M}^{+}(\Gamma): F(s, y)=0, s \leq \log x_{1}\right\} \\
\mathcal{M}_{x_{1}}^{-}(\Gamma)=\left\{F \in \mathcal{M}^{-}(\Gamma): F(s, y)=0, s \geq-\log x_{1}\right\}
\end{gathered}
$$

and let

$$
\begin{gathered}
\mathcal{P}_{x_{1}}^{+}: \mathcal{M}^{+}(\Gamma) \longrightarrow \mathcal{M}_{x_{1}}^{+}(\Gamma), \text { and } \\
\mathcal{P}_{x_{1}}^{-}: \mathcal{M}^{-}(\Gamma) \longrightarrow \mathcal{M}_{x_{1}}^{-}(\Gamma)
\end{gathered}
$$

denote the orthogonal projections with respect to the norm $\mathcal{N}$ defined in (3.2). Since $\mathcal{M}^{ \pm}(\Gamma)$ and $\mathcal{M}_{x_{1}}^{ \pm}(\Gamma)$ are determined by $\left.\mathcal{S}\right|_{\mathbb{R} \times \Gamma}$, the projections $\mathcal{P}_{x_{1}}^{ \pm}$are also determined by $\left.\mathcal{S}\right|_{\mathbb{R} \times \Gamma}$.

In view of finite speed of propagation and Theorem 2.2

$$
\begin{aligned}
& \mathcal{M}_{x_{1}}^{+}(\Gamma)=\left\{\left.\mathcal{R}_{+}(0, h)\right|_{\mathbb{R} \times \Gamma}: h \in L_{a c}^{2}(X), h(z)=0, z \in \mathcal{D}_{\log x_{1}}(\Gamma)\right\}, \\
& \mathcal{M}_{x_{1}}^{-}(\Gamma)=\left\{\left.\mathcal{R}_{-}(0, h)\right|_{\mathbb{R} \times \Gamma}: h \in L_{a c}^{2}(X), h(z)=0, z \in \mathcal{D}_{\log x_{1}}(\Gamma)\right\} .
\end{aligned}
$$

As in [37], the key to proving Proposition 4.1 is to understand the effect of the projectors $\mathcal{P}_{x_{1}}^{ \pm}$ on the initial data. First we deal with the case of no eigenvalues. In this case, $L^{2}(X)=L_{a c}^{2}(X)$.

Lemma 4.2. Let $(X, g)$ be an asymptotic hyperbolic manifold such that $\Delta_{g}$ has no eigenvalues. Let $x$ be such that (1.3) holds in $(0, \epsilon) \times \partial X$. For $x_{1} \in(0, \epsilon)$, let $\mathcal{P}_{x_{1}}^{-}$denote the orthogonal projector defined in (4.3). Let $\chi_{x_{1}}$ be the characteristic function of the set $X_{x_{1}}=X \backslash \mathcal{D}_{\log x_{1}}(\Gamma)$. Then for every $f \in L_{a c}^{2}(X)=L^{2}(X)$,

$$
\mathcal{P}_{x_{1}}^{-}\left(\left.\mathcal{R}_{-}(0, f)\right|_{\mathbb{R} \times \Gamma}\right)=\left.\mathcal{R}_{-}\left(0, \chi_{x_{1}} f\right)\right|_{\mathbb{R} \times \Gamma}
$$

Proof. Then since $\mathcal{P}_{x_{1}}^{-}$is a projector, there exists $f_{x_{1}} \in L^{2}(X)$ such that $\mathcal{P}_{x_{1}}^{-}\left(\left.\mathcal{R}_{-}(0, f)\right|_{\mathbb{R} \times \Gamma}\right)=$ $\left.\mathcal{R}_{-}\left(0, f_{x_{1}}\right)\right|_{\mathbb{R} \times \Gamma}$, and for every $h \in L^{2}(X)$ supported in $X_{x_{1}}$,

$$
\left\langle\left.\mathcal{R}_{-}\left(0, f_{x_{1}}\right)\right|_{\mathbb{R} \times \Gamma},\left.\mathcal{R}_{-}(0, h)\right|_{\mathbb{R} \times \Gamma}\right\rangle_{\mathcal{N}}=\left\langle f_{x_{1}}, h\right\rangle_{L^{2}(X)}=\langle f, h\rangle_{L^{2}(X)} .
$$

Hence $f_{x_{1}}=\chi_{x_{1}} f$. 
Next we analyze the singularities of the wave which produces $\mathcal{R}_{ \pm}\left(0, \chi_{x_{1}} f\right)$. Notice that if $f=0$ in $\mathcal{D}_{\log x_{1}}(\Gamma)$, and if $x \leq x_{1}$ and $y \in \Gamma$, then $d_{g}\left((x, y), \Gamma_{x_{1}}\right)=\log \left(\frac{x_{1}}{x}\right)$, and hence, $f(x, y)=0$.

If $F=\left.\mathcal{R}_{-}(0, f)\right|_{\mathbb{R} \times \Gamma}, f \in L^{2}(X)$, one can take $F$ and $f$ smooth by taking convolution with $\phi \in C_{0}^{\infty}$ even. Indeed, if $\psi_{1}$ such that $\hat{\phi}(\lambda)=\psi_{1}\left(\lambda^{2}\right)$, and $G=\phi * F=\left.\mathcal{R}_{-}(0, h)\right|_{\mathbb{R} \times \Gamma}$ with $h=\psi_{1}\left(\Delta_{g}-\frac{n^{2}}{4}\right) \mathcal{R}_{-}^{-1} F$. The point is that the singularities of $\chi_{x_{1}} f$ at $\Gamma_{x_{1}}$ produce the singularities of $\mathcal{R}_{+}\left(0, \chi_{x_{1}} f\right)$ at $\left(\log x_{1}, y\right), y \in \Gamma$. By expanding the solution to (1.11) with initial data, $\left(0, \chi_{x_{1}} f\right)$ we obtain,

Lemma 4.3. Let $x$ be a defining function of $\partial X$ such that (1.3) holds. Let $F \in \mathcal{M}^{-}(\Gamma)=$ $\left.\mathcal{R}_{-}(0, f)\right|_{\mathbb{R} \times \Gamma}$ with $F$ and $f$ smooth. Then there exists $\epsilon>0$ such that for any $x_{1} \in(0, \epsilon)$, any $F \in \mathcal{M}^{b}$, and for $\left|s-\log x_{1}\right|$ small enough,

$$
\mathcal{R}_{+} \mathcal{R}_{-}^{-1}\left(\mathcal{P}_{x_{1}}^{b} F\right)(s, y)=\frac{1}{2} x_{1}^{-n / 2} f\left(x_{1}, y\right) \frac{|h|^{1 / 4}\left(x_{1}, y\right)}{|h|^{1 / 4}(0, y)}\left(s-\log x_{1}\right)_{+}^{0}+\text { smoother terms, }
$$

We refer the reader to the proof of Lemma 8.9 of [37].

Notice that the left hand side of (4.4) is determined by $\left.\mathcal{S}\right|_{\mathbb{R} \times \Gamma}$, so the right hand side is also determined by $\left.\mathcal{S}\right|_{\mathbb{R} \times \Gamma}$. By assumption-since the scattering matrices are equal- $h_{0,1}=h_{0,2}$ on $\Gamma$. Therefore $\left|h_{1}\right|(0, y)=\left|h_{2}\right|(0, y), y \in \Gamma$ and we obtain the following result:

Corollary 4.4. Let $\left(X_{1}, g_{1}\right)$ and $\left(X_{2}, g_{2}\right)$ be asymptotically hyperbolic manifolds satisfying the hypothesis of Theorem 1.3. Moreover, assume that $\Delta_{g_{j}}, j=1,2$, have no eigenvalues. Let $\mathcal{R}_{j, \pm}$, $j=1,2$, denote the corresponding forward or backward radiation fields defined in coordinates in which (1.3) holds. Then there exists an $\epsilon>0$ such that, for $(x, y) \in[0, \epsilon) \times \Gamma$,

$$
\begin{array}{ll}
\left|h_{1}\right|^{1 / 4}(x, y) \mathcal{R}_{1,-}^{-1} F(x, y)=\left|h_{2}\right|^{1 / 4}(x, y) \mathcal{R}_{2,-}^{-1} F(x, y), & \forall F \in \mathcal{M}^{-}(\Gamma), \\
\left|h_{1}\right|^{1 / 4}(x, y) \mathcal{R}_{1,+}^{-1} F(x, y)=\left|h_{2}\right|^{1 / 4}(x, y) \mathcal{R}_{2,+}^{-1} F(x, y), & \forall F \in \mathcal{M}^{+}(\Gamma) .
\end{array}
$$

Proposition 4.1 easily follows from this result. Indeed, since

$$
\mathcal{R}_{j,-}^{-1}\left(\frac{\partial^{2}}{\partial s^{2}} F\right)=\left(\Delta_{g_{j}}-\frac{n^{2}}{4}\right) \mathcal{R}_{j,-}^{-1} F .
$$

if we apply Corollary 4.4 to $\partial_{s}^{2} F$, we obtain

$$
\left|h_{1}\right|^{1 / 4}(x, y)\left(\Delta_{g_{1}}-\frac{n^{2}}{4}\right) \mathcal{R}_{1,-}^{-1} F(x, y)=\left|h_{2}\right|^{1 / 4}(x, y)\left(\Delta_{g_{2}}-\frac{n^{2}}{4}\right) \mathcal{R}_{2,-}^{-1} F(x, y) .
$$

Set $\mathcal{R}_{1,-}^{-1} F=f$. Since $F$ is arbitrary and the metrics have no eigenvalues, the equations (4.5) and (4.7) give

$$
\left|h_{1}\right|^{1 / 4}(x, y)\left(\Delta_{g_{1}}-\frac{n^{2}}{4}\right) f(x, y)=\left|h_{2}\right|^{1 / 4}(x, y)\left(\Delta_{g_{2}}-\frac{n^{2}}{4}\right) \frac{\left|h_{1}\right|^{1 / 4}(x, y)}{\left|h_{2}\right|^{1 / 4}(x, y)} f(x, y),
$$

for all $f \in C^{\infty}((0, \epsilon) \times \Gamma)$. Therefore the operators on both sides of (4.8) are equal. In particular, the coefficients of the principal parts of $\Delta_{g_{1}}$ are equal to those of $\Delta_{g_{2}}$, and hence the tensors $h_{1}$ and $h_{2}$ from (1.3) are equal. This proves that

$$
\mathcal{R}_{1,-}^{-1}\left(s, y, x^{\prime}, y^{\prime}\right)=\mathcal{R}_{2,-}^{-1}\left(s, y, x^{\prime}, y^{\prime}\right), \quad y, y^{\prime} \in \Gamma, \quad x^{\prime} \in[0, \varepsilon),
$$

and of course the same holds for the forward radiation field. Since $\mathcal{R}_{ \pm}$are unitary, $\mathcal{R}_{ \pm}^{-1}=\mathcal{R}_{ \pm}^{*}$, and hence this determines the kernel of $\mathcal{R}_{ \pm}$. This proves Proposition (4.1) in the case of no eigenvalues. 
Now we remove the assumption that there are no eigenvalues. The only poles of the resolvent

$$
R\left(\frac{n}{2}+i \lambda\right)=\left(\Delta_{g}-\frac{n^{2}}{4}-\lambda^{2}\right)^{-1} \text { in }\{\Im \lambda<0\}
$$

correspond to the finitely many eigenvalues of $\Delta_{g}$. Proposition 3.6 of [12] states that if $\lambda_{0} \in i \mathbb{R}_{-}$ is such that $n^{2} / 4+\lambda_{0}^{2}$ is an eigenvalue of $\Delta_{g}$, then the scattering matrix has a pole at $\lambda_{0}$ and its residue is given by

$$
\operatorname{Res}_{\lambda_{0}} A(\lambda)=\left\{\begin{array}{l}
\Pi_{\lambda_{0}}, \quad \text { if }-i \lambda_{0} \notin \frac{\mathbb{N}}{2}, \\
\Pi_{\lambda_{0}}-P_{l}, \quad \text { if }-i \lambda_{0}=\frac{l}{2}, \quad l \in \mathbb{N},
\end{array}\right.
$$

where $P_{l}$ is a differential operator whose coefficients depend on derivatives of the tensor $h$ at $\partial X$, and the Schwartz kernel of $\Pi_{\lambda_{0}}$ is

$$
K\left(\Pi_{\lambda_{0}}\right)\left(y, y^{\prime}\right)=-2 i \lambda_{0} \sum_{j=1}^{N_{0}} \phi_{j}^{0} \otimes \phi_{j}^{0}\left(y, y^{\prime}\right),
$$

where $\phi_{j}^{0}(y)$ is defined by

$$
\phi_{j}^{0}(y)=\left.x^{-n / 2-\lambda_{0}} \phi_{j}(x, y)\right|_{x=0} .
$$

Here $N_{0}$ is the multiplicity of the eigenvalue $n^{2} / 4+\lambda_{0}^{2}$, and $\phi_{j}, 1 \leq j \leq N_{0}$, are the corresponding orthonormalized eigenfunctions.

Since $A_{1, \Gamma}=A_{2, \Gamma}, \lambda \in \mathbb{R} \backslash 0$, it follows from Theorem 1.1 that in coordinates where (4.1) is satisfied, all derivatives of $h_{1}$ and $h_{2}$ agree at $x=0$ on $\Gamma$. Therefore the operators $P_{l}$ in (4.9) are the same in $\Gamma$. Then (4.9), (4.10), and the meromorphic continuation of the scattering matrix show that $\Delta_{g_{1}}$ and $\Delta_{g_{2}}$ have the same eigenvalues with the same multiplicity. Moreover, (4.10) implies that if $\phi_{j}$ and $\psi_{j}, 1 \leq j \leq N_{0}$, are orthonormal sets of eigenfunctions of $\Delta_{g_{1}}$ and $\Delta_{g_{2}}$, respectively, corresponding to the eigenvalue $n^{2} / 4+\lambda_{0}^{2}$, then there exists a constant orthogonal $\left(N_{0} \times N_{0}\right)$-matrix $A$ such that $\left.\Phi^{0}\right|_{\Gamma}=\left.A \Psi^{0}\right|_{\Gamma}$, where $\left(\Phi^{0}\right)^{T}=\left(\phi_{1}^{0}, \phi_{2}^{0}, \ldots, \phi_{N_{0}}^{0}\right)$ and $\left(\Psi^{0}\right)^{T}=\left(\psi_{1}^{0}, \psi_{2}^{0}, \ldots, \psi_{N_{0}}^{0}\right)$. So by redefining one set of eigenfunctions from, let us say, $\Psi$ to $A \Psi$, where $\Psi^{T}=\left(\psi_{1}, \psi_{2}, \ldots, \psi_{N_{0}}\right)$, we may assume that

$$
\phi_{j}^{0}(y)=\psi_{j}^{0}(y), \quad y \in \Gamma \quad j=1,2, . ., N_{0} .
$$

Note that this does not change the orthonormality of the eigenfunctions in $X_{2}$ because $A$ is orthogonal. Denote the eigenvalues of $\Delta_{g_{1}}$ and $\Delta_{g_{2}}$, which we know are equal, by

$$
\mu_{j}=\frac{n^{2}}{4}+\lambda_{j}^{2}, \quad \lambda_{j} \in i \mathbb{R}_{-}, \quad 1 \leq j \leq N
$$

They are also ordered so that $\mu_{1} \leq \mu_{2} \leq \cdots \leq \mu_{N}$.

Again, we use that the singularities of $\chi_{x_{1}} f$ at $\Gamma_{x_{1}}$ produce the singularities of $\mathcal{R}_{+}\left(0, \chi_{x_{1}} f\right)$ at $\left(\log x_{1}, y\right), y \in \Gamma$ and expand the solution to (1.11) with initial data, $\left(0, \chi_{x_{1}} f\right)$. However, in this case $L^{2}(X) \neq L_{a c}^{2}(X)$ and hence Lemma 4.2 is not valid, and we have to replace it by the following

Lemma 4.5. Let $(X, g)$ be an asymptotic hyperbolic manifold and let $\phi_{j}, 1 \leq j \leq N$, denote the orthonormal set of eigenfunctions of $\Delta_{g}$. Let $x$ be such that (1.3) holds in $(0, \epsilon) \times \partial X$. For $x_{1} \in(0, \epsilon)$, let $\mathcal{P}_{x_{1}}^{-}$denote the orthogonal projector defined in (4.3). Let $\chi_{x_{1}}$ be the characteristic function of the set $X_{x_{1}}=X \backslash \mathcal{D}_{\log x_{1}}(\Gamma)$. There exists $\varepsilon_{0}$ such that if $\varepsilon<\varepsilon_{0}$, then for every 
$f \in L_{a c}^{2}(X)$ there exist $\alpha\left(x_{1}, f\right)$, which is a linear function of $f$, such that

$$
\mathcal{P}_{x_{1}}^{-}\left(\left.\mathcal{R}_{-}(0, f)\right|_{\mathbb{R} \times \Gamma}\right)=\left.\mathcal{R}_{-}\left(0, \chi_{x_{1}}\left(f-\sum_{j=1}^{N} \alpha_{j}\left(x_{1}, f\right) \phi_{j}\right)\right)\right|_{\mathbb{R} \times \Gamma} .
$$

Proof. Let $h \in L_{a c}^{2}(X)$ be supported in $X_{x_{1}}$. Then since $\mathcal{P}_{x_{1}}^{-}$is a projector, there exists $f_{x_{1}} \in$ $L_{a c}^{2}(X)$, supported in $X_{x_{1}}$, such that $\mathcal{P}_{x_{1}}^{-}\left(\left.\mathcal{R}_{-}(0, f)\right|_{\mathbb{R} \times \Gamma}\right)=\left.\mathcal{R}_{-}\left(0, f_{x_{1}}\right)\right|_{\mathbb{R} \times \Gamma}$, and for every $h \in$ $L_{a c}^{2}(X)$ supported in $X_{x_{1}}$,

$$
\left\langle\left.\mathcal{R}_{-}\left(0, f_{x_{1}}\right)\right|_{\mathbb{R} \times \Gamma},\left.\mathcal{R}_{-}(0, h)\right|_{\mathbb{R} \times \Gamma}\right\rangle_{\mathcal{N}}=\left\langle f_{x_{1}}, h\right\rangle_{L^{2}(X)}=\langle f, h\rangle_{L^{2}(X)} .
$$

Hence $\left\langle\left(f_{x_{1}}-f\right), h\right\rangle=0$ for all $h \in C_{0}^{\infty}(X) \cap L_{a c}^{2}(X)$ supported in $X_{1}$. Therefore, $f_{x_{1}}-f=$ $\chi_{x_{1}} \sum_{j=1}^{N} \alpha_{j} \phi_{j}$. Since $f_{x_{1}} \in L_{a c}^{2}(X),\left\langle f_{x_{1}}, \chi_{x_{1}} \phi_{j}\right\rangle=0$, and hence

$$
\left\langle f, \chi_{x_{1}} \phi_{k}\right\rangle=\sum_{j=1}^{N} \alpha_{j}\left\langle\chi_{x_{1}} \phi_{j}, \chi_{x_{1}} \phi_{k}\right\rangle .
$$

This gives a linear system of equations

$$
\begin{gathered}
M \alpha=F, \quad \alpha^{T}=\left(\alpha_{1}, \ldots, \alpha_{N}\right), \quad F^{T}=\left(F_{1}\left(x_{1}\right), \ldots, F_{N}\left(x_{1}\right)\right\}, \\
M_{j k}\left(x_{1}\right)=\left\langle\chi_{x_{1}} \phi_{j}, \chi_{x_{1}} \phi_{k}\right\rangle, \quad F_{k}\left(x_{1}\right)=\left\langle f, \chi_{x_{1}} \phi_{k}\right\rangle .
\end{gathered}
$$

Since eigenfunctions are orthonormal, then for $x_{1}=0, M_{j k}(0)=\delta_{j k}$. Therefore the system has a solution if $x_{1}<\varepsilon_{0}$, for certain $\varepsilon_{0}$, which does not depend of $f$. Notice that, since $f \in L_{a c}^{2}(X)$, then for $x_{1}=0, F_{k}(0)=0$, and hence, $\alpha(0, f)=0$.

As in [37, we shall denote

$$
T\left(x_{1}\right) f=\sum_{j} \alpha_{j}\left(x_{1}, f\right) \phi_{j}
$$

Since $\alpha(0, f)=0, T(0)=0$. Therefore one can pick $\varepsilon$ small so that

$$
\left\|T\left(x_{1}\right)\right\|<\frac{1}{2} \text { for } x_{1}<\varepsilon .
$$

In the case, Lemma 4.3 and Corollary 4.4 have to be substituted by

Lemma 4.6. Let $(X, g)$ be an asymptotically hyperbolic manifold, let $\phi_{j}, 1 \leq j \leq N$, denote the eigenfunctions of $\Delta_{g}$. Let $x$ be a defining function of $\partial X$ such that (1.3) holds, let $T\left(x_{1}\right)$ be defined as above and let $F \in \mathcal{M}^{-}(\Gamma), F=\left.\mathcal{R}_{-}(0, f)\right|_{\mathbb{R} \times \Gamma}$ with $F$ and $f$ smooth. Then there exists $\epsilon>0$ such that for any $x_{1} \in(0, \epsilon)$, and for $\left|s-\log x_{1}\right|$ small enough,

$$
\mathcal{R}_{+} \mathcal{R}_{-}^{-1}\left(\mathcal{P}_{x_{1}}^{b} F\right)(s, y)=\frac{1}{2} x_{1}^{-n / 2}\left[\left(\operatorname{Id}-T\left(x_{1}\right)\right) f\right]\left(x_{1}, y\right) \frac{|h|^{1 / 4}\left(x_{1}, y\right)}{|h|^{1 / 4}(0, y)}\left(s-\log x_{1}\right)_{+}^{0}+\text { smoother terms, }
$$

Corollary 4.7. Let $\left(X_{1}, g_{1}\right)$ and $\left(X_{2}, g_{2}\right)$ be asymptotically hyperbolic manifolds satisfying the hypothesis of Theorem 1.3. Let $\mathcal{R}_{j, \pm}, j=1,2$, denote the corresponding forward or backward 
radiation fields defined in coordinates in which (1.3) holds. Then there exists an $\epsilon>0$ such that, for $(x, y) \in[0, \epsilon) \times \Gamma$,

$$
\begin{array}{ll}
\left|h_{1}\right|^{1 / 4}(x, y)\left(\operatorname{Id}-T_{1}(x)\right) \mathcal{R}_{1,-}^{-1} F(x, y)=\left|h_{2}\right|^{1 / 4}(x, y) \mathcal{R}_{2,-}^{-1}\left(\operatorname{Id}-T_{2}(x)\right) F(x, y), & \forall F \in \mathcal{M}^{-}(\Gamma), \\
\left|h_{1}\right|^{1 / 4}(x, y)\left(\operatorname{Id}-T_{1}(x)\right) \mathcal{R}_{1,+}^{-1} F(x, y)=\left|h_{2}\right|^{1 / 4}(x, y)\left(\operatorname{Id}-T_{2}(x)\right) \mathcal{R}_{2,+}^{-1} F(x, y), & \forall F \in \mathcal{M}^{+}(\Gamma) .
\end{array}
$$

Now, as in the case of no eigenvalues, we use (4.15) to show that we can apply the same identity to $\left(\Delta_{g_{j}}-\frac{n^{2}}{4}\right) f_{j}$, instead of $f_{j}$ and so we have

$$
\left|h_{1}\right|^{\frac{1}{4}}\left(\operatorname{Id}-T_{1}(x)\right)\left(\Delta_{g_{1}}-\frac{n^{2}}{4}\right) f_{1}(x, y)=\left|h_{2}\right|^{\frac{1}{4}}\left(\operatorname{Id}-T_{2}(x)\right)\left|h_{2}\right|^{\frac{1}{4}}\left(\Delta_{g_{2}}-\frac{n^{2}}{4}\right) f_{2}(x, y) .
$$

If we denote $\mathcal{R}_{j,-}^{-1} F(x, y)=f_{j}(x, y)$, and pick $\varepsilon$ small so that (4.13) holds, we deduce from (4.15) that if $(x, y) \in[0, \varepsilon) \times \Gamma$,

$$
\begin{gathered}
f_{2}(x, y)=\left(\operatorname{Id}-T_{2}(x)\right)^{-1}\left(\operatorname{Id}-T_{1}(x)\right) \frac{\left|h_{1}\right|^{\frac{1}{4}}}{\left|h_{2}\right|^{\frac{1}{4}}} f_{1}(x, y)=\frac{\left|h_{1}\right|^{\frac{1}{4}}}{\left|h_{2}\right|^{\frac{1}{4}}} f_{1}(x, y)+K(x) f_{1}(x, y), \\
\left(\Delta_{g_{2}}-\frac{n^{2}}{4}\right) f_{2}(x, y)=\left(\operatorname{Id}-T_{2}(x)\right)^{-1}\left(\operatorname{Id}-T_{1}(x)\right) \frac{\left|h_{1}\right|^{\frac{1}{4}}}{\left|h_{2}\right|^{\frac{1}{4}}}\left(\Delta_{g_{1}}-\frac{n^{2}}{4}\right) f_{1}(x, y)= \\
\frac{\left|h_{1}\right|^{\frac{1}{4}}}{\left|h_{2}\right|^{\frac{1}{4}}}\left(\Delta_{g_{1}}-\frac{n^{2}}{4}\right) f_{1}(x, y)+\widetilde{K} f_{1}(x) .
\end{gathered}
$$

where $K$ and $\widetilde{K}$ are compact operators. If one substitutes the first equation into the second, one obtains

$$
\frac{\left|h_{1}\right|^{\frac{1}{4}}}{\left|h_{2}\right|^{\frac{1}{4}}}\left(\Delta_{g_{1}}-\frac{n^{2}}{4}\right) f_{1}(x, y)-\left(\Delta_{g_{2}}-\frac{n^{2}}{4}\right) \frac{\left|h_{1}\right|^{\frac{1}{4}}}{\left|h_{2}\right|^{\frac{1}{4}}} f_{1}(x, y)=\mathcal{K} f_{1},
$$

where $\mathcal{K}$ is a compact operator. Since the operator on the left hand side is a differential operator, and the operator on the right hand side is compact, they both must be equal to zero. As above, we conclude that in coordinates $(x, y)$, the coefficients of the operators $\Delta_{g_{1}}$ are equal to those of $\Delta_{g_{2}}$. Hence we must have $h_{1}(x, y, d y)=h_{2}(x, y, d y)$.

We still have to show (4.2) holds in the case where eigenvalues exist. Let $F \in \mathcal{M}^{+}(\Gamma)$, and let $f_{j}=\mathcal{R}_{j,+}^{-} F$. Let $v_{j}$ satisfy (1.11) with initial data $\left(0, f_{j}\right)$. Since $\Delta_{g_{1}}=\Delta_{g_{2}}$ in $(0, \varepsilon) \times \Gamma$, we have $P\left(v_{1}-v_{2}\right)=0$, where $P$ is defined in (2.2). Then by the proof of Theorem 2.2, we have $f_{1}=f_{2}$ in $(0, \varepsilon) \times \Gamma$. Since $F$ is arbitrary, (4.2) follows. This ends the proof of Proposition 4.1.

Finally, we will prove Theorem 1.3. The fact that the metrics are equal in these coordinates implies that there exist $V_{j, \varepsilon} \subset X_{j}, j=1,2$, neighborhoods of $\Gamma$ and $C^{\infty}$ differomomrphisms

$$
\Psi_{j, \varepsilon}: \Gamma \times[0, \varepsilon) \longrightarrow V_{j, \varepsilon} \subset X_{j}
$$

such that

$$
\Psi_{1, \epsilon}^{*}\left(\left.g_{1}\right|_{V_{1, \epsilon}}\right)=\Psi_{2, \epsilon}^{*}\left(\left.g_{2}\right|_{V_{2, \epsilon}}\right) .
$$

Therefore, the map

$$
\Psi_{\varepsilon}: V_{1, \varepsilon} \longrightarrow V_{2, \varepsilon}, \text { defined by } \Psi_{\varepsilon}=\Psi_{1, \varepsilon} \circ \Psi_{2, \varepsilon}^{-1},
$$

satisfies 


$$
\Psi_{\varepsilon}^{*}\left(\left.g_{2}\right|_{U_{2, \epsilon}}\right)=\left.g_{1}\right|_{U_{1, \epsilon}} \text {, and } \Psi_{\varepsilon}=\operatorname{Id} \text { on } \Gamma .
$$

We will show that $\Psi_{\varepsilon}$ can be extended to the whole manifolds, and to do that we will show that the fact that scattering matrices are equal at $\Gamma$ implies that there exist compact manifolds with boundary $X_{j, \varepsilon} \subset X_{j}$ such that $\Gamma_{\varepsilon} \subset X_{1, \varepsilon} \cap X_{2, \varepsilon}$ as manifolds and that the eigenvalues of $\Delta_{g_{j}}$ with Dirichlet data on $X_{j, \varepsilon}$ are equal and the traces of the normal derivatives of the Dirichlet eigenfunctions of $\Delta_{g_{j}}, j=1,2$ coincide at $\Gamma$. We shall then use the following result due to Kurylev and Lassas [24] and [26].

Proposition 4.8. Assume that $\left(Z_{1}, g_{1}\right)$ and $\left(Z_{2}, g_{2}\right)$ are smooth Riemannian manifolds with boundary $\partial Z_{1}$ and $\partial Z_{2}$, respectively. Assume that there are open sets $\Gamma_{1} \subset \partial Z_{1}$ and $\Gamma_{2} \subset \partial Z_{2}$, such that the boundary spectral data on $\Gamma_{2}$ and $\Gamma_{2}$ coincide. Namely, if $\alpha_{1, j}$, and $\varphi_{1, j}$ are the eigenvalues and the normalized Dirichlet eigenfunctions of $\Delta_{g_{1}}$ and and $\alpha_{2, j}, \varphi_{2, j}$ are the eigenvalues and the normalized Dirichlet eigenfunctions of $\Delta_{g_{2}}$,

$$
\Gamma_{1}=\Gamma_{2} \text { as manifolds, and } \alpha_{1, j}=\alpha_{2, j},\left.\quad \partial_{\nu} \varphi_{1, j}\right|_{\Gamma_{1}}=\left.\partial_{\nu} \tilde{\varphi}_{2, j}\right|_{\Gamma_{2}}, \quad j=1,2, \ldots
$$

Then there exists a diffeomorphism $\Psi: Z_{1} \longrightarrow Z_{2}$ such that $\Psi^{*} \tilde{g}=g$ and $\Psi=\operatorname{Id}$ on $\Gamma$.

Note that $X_{j, \epsilon}=X_{j} \backslash\left\{x_{j}<\epsilon\right\}$ are smooth compact manifolds with boundary and that there are open sets $\Gamma_{1, \epsilon} \subset \partial X_{1, \epsilon}, \Gamma_{2, \epsilon} \subset \partial X_{2, \epsilon}$ which can be identified by the diffeomorphisms $\Psi_{j, \varepsilon}$ with $\Gamma \times\{\epsilon\}=\Gamma_{\epsilon}$. We think of $\Gamma_{\epsilon}$ as an open subset of the boundary smooth compact manifolds $X_{j, \varepsilon}$. We have shown that the fact that the two metrics have the same scattering matrix restricted to $\Gamma$, implies that $\Delta_{g_{1}}=\Delta_{g_{2}}$ in $[0, \varepsilon) \times \Gamma$. The next step is to show that $\left(X_{j, \varepsilon}, g_{j}\right), j=1,2$, have the same spectral data in $\Gamma_{\epsilon}$. Then Proposition (4.8) shows that for $\varepsilon>0$ fixed, there exists a $C^{\infty}$ diffeomorphism $\widetilde{\Psi}_{\varepsilon}$, such that

$$
\begin{gathered}
\widetilde{\Psi}_{\varepsilon}: \bar{X}_{1, \varepsilon} \longrightarrow \bar{X}_{2, \varepsilon},\left.\quad \widetilde{\Psi}_{\varepsilon}\right|_{\Gamma_{\varepsilon}}=\mathrm{Id}, \\
\Psi_{\varepsilon}^{*}\left(\left.g_{2}\right|_{\bar{X}_{2, \varepsilon}}\right)=\left.g_{1}\right|_{\bar{X}_{1, \varepsilon}} .
\end{gathered}
$$

Now one has to extend this diffeomorphism up to $X_{1}$. Given a point $p \in X_{1} \backslash X_{1, \varepsilon}$ take the integral curve of $\nabla_{x_{1}^{2} g_{1}} x_{1}$ starting at $p$ and flow along this curve until it hits $p_{\varepsilon} \in \partial X_{1, \varepsilon}$ at time $s(p)$. In other words, $p_{\varepsilon}=\exp \left(s(p) \nabla_{x_{1}^{2} g_{1}} x_{1}\right)(p)$. Let $q_{\varepsilon}=\Psi_{\varepsilon}\left(p_{\varepsilon}\right) \in \partial X_{2, \varepsilon}$. In view of (4.20), and since $g_{j}=\frac{d x_{j}^{2}}{x_{j}^{2}}+\frac{h_{j}(x, y, d y)}{x_{j}^{2}}, j=1,2$, near $\partial X_{j, \varepsilon}$, it follows that $\left.d \Psi_{\varepsilon}\left(\nabla_{x_{1}^{2} g_{1}} x_{1}\right)\right|_{\partial X_{2, \varepsilon}}=\left.\nabla_{x_{2}^{2} g_{2}} x_{2}\right|_{\partial X_{2, \varepsilon}}$. Now follow the integral curve of $\nabla_{x_{2}^{2} g_{2}} x_{2}$ starting at $q_{\varepsilon}$, and flow $s(p)$ units along this curve away from $X_{2, \varepsilon}$. In other words, in $X_{j} \backslash X_{j, \varepsilon}$ the map $\widetilde{\Psi}_{\varepsilon}$ is given by

$$
\exp \left(-s(p) \nabla_{x_{2}^{2} g_{2}} x_{2}\right) \circ \Psi_{\varepsilon} \circ \exp \left(s(p) \nabla_{x_{1}^{2} g_{1}} x_{1}\right)=\widetilde{\Psi}_{\varepsilon} .
$$

This gives a map,

$$
\widetilde{\Psi}: X_{1} \longrightarrow X_{2},\left.\widetilde{\Psi}\right|_{\Gamma}=\mathrm{Id} \text { and that } \widetilde{\Psi}^{*} g_{2}=g_{1} .
$$

To prove that $\left(X_{j, \varepsilon}, \Delta_{g_{j}}\right)$ have the same boundary data at $\Gamma_{\varepsilon}$, we take Fourier transform of $\mathcal{R}_{ \pm}$ in the variable $s$. We know from (4.2) that if $E_{j}\left(\frac{n}{2}+i \lambda, y^{\prime}, z\right)=\widehat{\mathcal{R}_{j,+}}(s, y, z)$, then

$$
E_{1}\left(\frac{n}{2}+i \lambda, y^{\prime}, z\right)=E_{2}\left(\frac{n}{2}+i \lambda, y^{\prime}, z\right), \quad y \in \Gamma, \quad z=(x, y) \in[0, \varepsilon) \times \Gamma .
$$




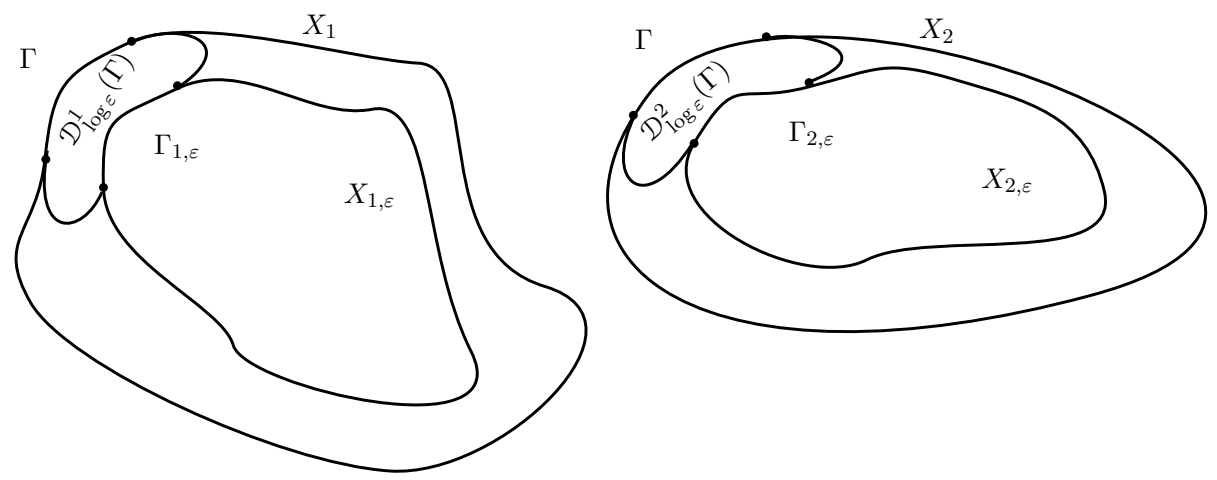

Figure 7 . The domains $X_{j, \varepsilon}, \mathcal{D}_{\log \varepsilon}^{j}(\Gamma)$ and the sets $\Gamma_{j, \varepsilon}$.

Using an argument due to Melrose, Lemma 3.2 of [35], Sá Barreto showed that for any $\lambda \neq 0$, the set of functions given by

$$
v_{j}(z, \lambda)=\int_{\partial X_{j}} E_{j}\left(\frac{n}{2}+i \lambda, y^{\prime}, z\right) \phi\left(y^{\prime}\right) d \operatorname{vol}_{h_{0}}\left(y^{\prime}\right), \quad j=1,2, \quad \phi \in C^{\infty}\left(\partial X_{j}\right),
$$

is dense in the set of solutions of

$$
\left(\Delta_{g_{j}}-\lambda^{2}-\frac{n^{2}}{4}\right) u=0 \quad \text { in } X_{j, \epsilon}, \quad j=1,2,
$$

in the Sobolev space $H^{k}\left(X_{j, \epsilon}\right)$ for any $k \geq 2$.

We know from (4.22) that $E_{1}\left(n / 2+i \lambda, y^{\prime}, x, y\right)=E_{2}\left(\frac{n}{2}+i \lambda, y^{\prime}, x, y\right)$ provided $x \in[0, \varepsilon)$ and $y, y^{\prime} \in \Gamma$, and hence $v_{1}(x, y, \lambda)=v_{2}(x, y, \lambda),(x, y) \in(0, \varepsilon) \times \Gamma, \lambda \in \mathbb{R} \backslash\{0\}$. Therefore their traces and normal derivatives at $\Gamma_{\epsilon}$ are equal at $\Gamma_{\varepsilon}$, and the density of this set implies that the same is true for solutions of (4.24).

We now recall that the graph of the Calderón projector of $\Delta_{g_{j}}-\frac{n^{2}}{4}-\lambda^{2}$ in $X_{j, \epsilon}, j=1,2$, restricted to $\Gamma$, which we denote by $C_{j, \lambda}$, is the closed subspace of $L^{2}(\Gamma) \times H^{1}(\Gamma)$ consisting of $(f, g) \in L^{2}(\Gamma) \times H^{1}(\Gamma)$ such that there exists $u$ satisfying

$$
\begin{gathered}
\left(\Delta_{g_{j}}-\frac{n^{2}}{4}-\lambda^{2}\right) u=0 \quad \text { in } X_{j, \epsilon}, \quad j=1,2, \\
\left.u\right|_{\partial X_{j, \epsilon}}=f \in C_{0}^{\infty}(\Gamma),\left.\quad \partial_{\nu} u\right|_{\Gamma}=g .
\end{gathered}
$$

Therefore $\lambda^{2}$ is in the Dirichlet spectrum of $\Delta_{g_{j}}-\frac{n^{2}}{4}$ in $X_{j, \epsilon}$ if and only if $C_{j, \lambda}$ contains a subspace of pairs of the form $(0, g), g \neq 0$. If $\lambda \in \mathbb{R}$, this only gives us eigenvalues greater than or equal to 0 . But since $E_{j}\left(\frac{n}{2}+i \lambda, y, z\right)$ are meromorphically in $\operatorname{Im} \lambda<0$, they must also agree in this region, and therefore we obtain all eigenvalues. Therefore we conclude that the spectral data $\left(\Delta_{g_{j}}, X_{j, \varepsilon}\right)$, $j=1,2$ coincide at $\Gamma$. Thus the hypothesis of Proposition (4.8) are satisfied, and hence the maps $\Psi_{\varepsilon}$ satisfyng (4.20) exist and this concludes the proof of Theorem 1.3 . 
RAPHAEL HORA AND ANTÔNIO SÁ BARRETO

\section{REFERENCES}

[1] M. Belishev and Y. Kurylev. To the reconstruction of a Riemannian manifold via its spectral data (BC-method). Comm. Partial Differential Equations 17 (1992), no. 5-6, 767-804.

[2] J-M. Bouclet. Absence of eigenvalue at the bottom of the continuous spectrum on asymptotically hyperbolic manifolds. arXiv:1208.0709

[3] C. Fefferman and C. R. Graham. Conformal invariants. Asterisque, vol. hors série, Soc. math. France, 1985, 95-116.

[4] F. G. Friedlander. Radiation Fields and hyperbolic scattering theory. Math. Proc. Camb. Phil. Soc., 88, 483-515, 1980

[5] F.G. Friedlander. Notes on the wave equation on asymptotically Euclidean manifolds. Journ. of Func. Anal.184, no.1, 1-18, (2001).

[6] C. Guillarmou. Meromorphic properties of the resolvent on asymptotically hyperbolic manifolds. Duke Math. Journal 129 (2005), no. 1., 1-37.

[7] C. Guillarmou and A. Sá Barreto. Scattering and inverse scattering on ACH manifolds. J. Reine Angew. Math. 622 (2008), 1-55.

[8] L. Guillopé. Fonctions zêta de Selberg et surfaces de géométrie finie. In Zeta Functions in Geometry (Tokyo, 1990), Adv. Stud. Pure Math. 21, Kinokuniya, Tokyo, 1992, 33-70.

[9] L. Guillopé and M. Zworski. Polynomial bounds on the number of resonances for some complete spaces of constant negative curvature near infinity. Asymptotic Anal. 11 (1995), 1-22.

[10] L. Guillopé and M. Zworski. Scattering asymptotics for Riemann surfaces. Ann. of Math. 145 (1997), 597-660.

[11] C.R. Graham. Volume and area renormalization for conformally compact Eistein metrics. The Proceedings of the 19th Winter School "Geometry and Physics" (Srní, 1999). Rend. Circ. Mat. Palermo (2) Suppl. No. 63 (2000), 31-42.

[12] C.R. Graham and M. Zworski. Scattering matrix in conformal geometry. Invent. Math., 152, 89-118, 2003.

[13] S. Helgason. The Radon transform on Euclidean spaces, compact two-point homogeneous spaces and Grassmann manifolds. Acta Math. 113, 153-18o (1965)

[14] S. Helgason. The Radon Transform. 2nd Edition Vol. 5, progress in mathematics, Birkhäuser, (1999)

[15] S. Helgason. Non-Euclidean analysis. Non-Euclidean geometries, 367Đ384, Math. Appl. (N. Y.), 581, Springer, New York, 2006.

[16] S. Helgason. The Abel, Fourier and Radon transforms on symmetric spaces. Indag. Math. (N.S.) 16 (2005), no. 3-4, 531Đ551.

[17] L. Hörmander. Uniqueness theorems for second order elliptic differential equations. Comm. Partial Differential Equations 8 (1983), no. 1, 21-64.

[18] L. Hörmander. Linear Partial Differential Operators. Springer Verlag, 1963.

[19] L. Hörmander. The Analysis of Linear Partial Differential Operators. vol. I-IV. Springer Verlag, 1994.

[20] L. Hörmander. On the uniqueness of the Cauchy problem under partial analyticity assumptions. Geometrical optics and related topics (Cortona, 1996), 179?219, Progr. Nonlinear Differential Equations Appl., 32, Birkh?user Boston, Boston, MA, 1997.

[21] M.S. Joshi and A. Sá Barreto.Recovering asymptotics of metrics from fixed energy scattering data. Inventiones Mathematicae 137, 127-143 (1999).

[22] M.S. Joshi and A. Sá Barreto. Inverse scattering on asymptotically hyperbolic manifold. Acta Mathematica Vol. 184, 41-86, (2000).

[23] M.S. Joshi and A. Sá Barreto. The Wave Group on Asymptotically Hyperbolic Manifolds. Journal of Functional Analysis, vol. 184, 291-312, (2001).

[24] A. Katchalov, Y. Kurylev, M. Lassas. Inverse Boundary Spectral Problems. Monographs and Surveys in Pure and Applied Mathematics 123, Chapman Hall/CRC-press, 2001, xi+290 pp.

[25] Y. Kurylev and M. Lassas. Hyperbolic inverse boundary-value problem and time continuation of the nonstationary Dirichlet-to-Neumann map. Proc. Royal. Soc. Edinburgh, 132A, 931-949, (2002)

[26] Y. Kurylev, M. Lassas. Hyperbolic inverse problem with data on a part of the boundary. AMS/IP Stud. Adv. Math. 16 (2000), 259-272, Amer. Math. Soc., Proceedings of Differential equations and mathematical physics (Birmingham, AL, 1999)

[27] P. Lax and R. Phillips. Translation representation for the solution of the non-Euclidean wave equation. Comm. Pure Appl. Math. 32 (1979), 617-667. 
[28] P. Lax and R. Phillips. Translation representation for automorphic solutions of the wave equation in nonEuclidean spaces I, II. Comm. Pure Appl. Math. 37, 303-328, (1984).

[29] P. Lax and R. Phillips. Translation representation for automorphic solutions of the wave equation in nonEuclidean spaces II. Comm. Pure Appl. Math. 37, 780-813, (1984).

[30] P. Lax and R. Phillips. A local Paley-Wiener theorem for the Radon transform of $L^{2}$ functions in a non-Euclidean setting. Comm. Pure Appl. Math. 35 no. 4, 531Đ554, (1982).

[31] P. Lax and R. Phillips. Scattering theory. Second edition. With appendices by Cathleen S. Morawetz and George Schmidt. Pure and Applied Mathematics, 26. Academic Press, Inc., Boston, MA, 1989.

[32] R. Mazzeo. The Hodge cohomology of a conformally compact metric. J. Differential Geom. 28 (1988), $309-339$.

[33] R. Mazzeo. Unique continuation at infinity and embedded eigenvalues for asymptotically hyperbolic manifolds. Amer. J. Math. 113 (1991), 25-45.

[34] R. Mazzeo and R. Melrose. Meromorphic extension of the resolvent on complete spaces with asymptotically constant negative curvature. J. Functional Analysis 75 (1987), 260-310.

[35] R. B. Melrose. Geometric scattering theory. Lectures at Stanford. Cambridge University Press, 1995.

[36] L. Robbiano and C. Zuily. Uniqueness in the Cauchy problem for operators with partially holomorphic coefficients. Invent. Math. 131 (1998), no. 3, $493 Đ 539$.

[37] A. Sá Barreto. Radiation fields, scattering and inverse scattering on asymptotically hyperbolic manifolds. Duke Math. Journal Vol 129, No. 3, 407-480, 2005.

[38] A. Sá Barreto and J. Wunsch. The radiation field is a Fourier integral operator. Ann. Inst. Fourier (Grenoble) 55 (2005), no. 1, 213-227.

[39] D. Tataru. Unique continuation for solutions to PDE's; between Hörmander's theorem and Holmgren's theorem. Comm. Partial Differential Equations 20 (1995), no. 5-6, 855-884.

[40] D. Tataru. Unique continuation for operators with partially analytic coefficients. J. Math. Pures Appl. 78, 505-521, (1999)

[41] G. Uhlmann. Developments in inverse problems since Calderón's foundational paper. Harmonic analysis and partial differential equations (Chicago, IL, 1996), 295-345, Chicago Lectures in Math., Univ. Chicago Press, Chicago, IL, 1999.

[42] A. Vasy and J. Wunsch. Absence of super-exponentially decaying eigenfunctions of Riemannian manifolds with pinched negative curvature. Math. Res. Lett. 12 (2005), no. 5-6, 673-684.

[43] A. Vasy. Analytic continuation and high energy estimates for the resolvent of the Laplacian on forms on asymptotically hyperbolic spaces. arXiv:1206.5454

E-mail address: rhora@math.purdue.edu

E-mail address: sabarre@math.purdue.edu

Department of Mathematics, Purdue University

150 North University Street, West Lafayette IN 47907, USA 